\title{
EXPLORING GTRS BASED RECOMMENDER SYSTEMS WITH USERS OF DIFFERENT RATING PATTERNS
}

\author{
A Thesis \\ Submitted to the Faculty of Graduate Studies and Research \\ In Partial Fulfillment of the Requirements \\ FOR THE DEGREE OF \\ MASTER OF SCIEnCE \\ IN \\ Computer Science \\ UNIVERSITY OF REGINA \\ By \\ Bingyu Li \\ Regina, Saskatchewan \\ July 2018
}

Copyright (c) 2018: Bingyu Li 


\section{UNIVERSITY OF REGINA}

\section{FACULTY OF GRADUATE STUDIES AND RESEARCH SUPERVISORY AND EXAMINING COMMITTEE}

Bingyu Li, candidate for the degree of Master of Science in Computer Science, has presented a thesis titled, Exploring GTRS Based Recommender Systems With Users

of Different Rating Patterns, in an oral examination held on Julyl 4, 2018. The following committee members have found the thesis acceptable in form and content, and that the candidate demonstrated satisfactory knowledge of the subject material.

External Examiner: $\quad$ Dr. Wei Peng, Faculty of Enginering \& Applied Science

Supervisor: $\quad$ Dr. JingTao Yao, Department of Computer Science

Committee Member: Dr. Sandra Zilles, Department of Computer Science

Committee Member: $\quad$ Dr. David Gerhard, Department of Computer Science

Chair of Defense: $\quad$ Dr. Allen Herman, Department of Mathematics \& Statistics 


\section{Abstract}

Recommender systems predict a new user's opinion on a collection of items by analyzing preference information of similar users. The Pawlak rough set model is one of the effective tools to make personalized recommendations. The game-theoretic rough set (GTRS) model improves the quality of the Pawlak rough set based recommendations by determining a pair of thresholds that could achieve a tradeoff between accuracy and coverage, which are two of the most prominent recommendation evaluation metrics. It should be noted that the performance of a recommendation algorithm may be affected by the rating patterns of the users in the considered dataset. The aim of this research is to evaluate how the performance of the Pawlak rough set based and the GTRS based recommendations vary on user groups that have different rating patterns. We conducted comparative experiments on five different data sam-

ples. The experimental results suggest that compared to the Pawlak rough set model, the GTRS model could not only obtain an improvement in coverage level, but also achieve an equal accuracy level on each of the considered data samples. In particular, it achieved a bigger advantage over the Pawlak rough set model on user groups that make a smaller number of rating records. This performance difference indicates that compared to the Pawlak rough set model, the GTRS model gives a better solution to make high quality personalized recommendations on datasets formed by users that make a smaller number of rating records. 


\section{Acknowledgments}

I would like to express my sincere gratitude to my supervisor, Dr. JingTao Yao, Professor of Computer Science, University of Regina, for the support, encouragement, and guidance he gave me in the thesis writing process.

I want to say a big thank you to other members of my committee, Dr. Sandra Zilles and Dr. David Gerhard, who have gave me so many helpful suggestions which resulted in an improved thesis. I would also like to thank Dr. Orland Hoeber, who helps me a lot in the formatting of my thesis.

The financial assistance provided by the Faculty of Graduate Studies and Research, and Natural Science and Engineering Council of Canada (NSERC) is very much appreciated.

I appreciate the help from everyone in our research group. From you I've learned things that I will carry with me for the rest of my life. I want to thank my wonderful friend, Gao and Conglian, for talking me through all the difficulties that I encountered in my research as well as in my personal life. And my sister Wendy, you have been the best accompany in my entire writing process, thank you so much for always having my back.

Finally, I want to express my gratitude to my parents, Zhi and Ying, who have always been so supportive for every decision that I made. Your constant encouragement is the best energy source to hold me through my graduate research. 


\section{Contents}

Abstract $\quad$ i

Table of Contents iii

$\begin{array}{lll}\text { Chapter } 1 & \text { Introduction } & 1\end{array}$

Chapter $2 \quad$ Background Knowledge and Related Work 4

Chapter 3 Pawlak Rough Set Based Recommendations 9

3.1 The Pawlak Rough Set Model . . . . . . . . . . . . . . . . . . . . . 9

3.2 The Probabilistic Rough Set Model . . . . . . . . . . . . . . . . . . 10

3.3 Pawlak Rough Set Based Recommendations . . . . . . . . . . . . . . 12

3.4 Accuracy and Coverage of the Pawlak Rough Set Based Recommen-

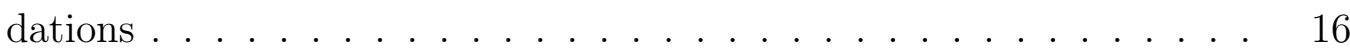

$\begin{array}{llr}\text { Chapter } 4 & \text { GTRS Based Recommendations } & 18\end{array}$

4.1 The Game-theoretic Rough Set Model . . . . . . . . . . . . . . . . . 18

4.2 Formulating a Two-player GTRS Based Game . . . . . . . . . . . . . 21

4.3 Formulating a Competitive Game between Accuracy and Coverage . . 22

4.4 Determining GTRS Thresholds with a Tradeoff between Accuracy and Coverage......................... 25 
$\begin{array}{lll}\text { Chapter } 5 & \text { Data Partitioning and Preprocessing }\end{array}$

5.1 Dataset . . . . . . . . . . . . . . . . . . 33

5.2 Data Partitioning . . . . . . . . . . . . . . . . . . . 33

5.3 Data Preprocessing . . . . . . . . . . . . . . . . . . 36

$\begin{array}{lll}\text { Chapter } 6 & \text { Statistical Analysis and Comparative Evaluation } & 38\end{array}$

6.1 Accuracy and Coverage Analysis . . . . . . . . . . . . . . 38

6.2 Accuracy and Coverage Comparison on Featured Data Samples .. 42

6.3 Decision Support Metric Analysis . . . . . . . . . . . . . . . . 48

6.3 .1 Precision-recall Analysis . . . . . . . . . . . . . . 50

6.3.2 Precision-recall Comparison on Featured Data Samples . . . . 53

6.3.3 Receiver Operating Curve Based Metrics Analysis . . . . . . . 54

6.3.4 ROC Curve-based Metrics Comparison on Featured Data Samples 55

6.4 Overall Performance Analysis and Comparison . . . . . . . . . . 57

$\begin{array}{lll}\text { Chapter } 7 & \text { Conclusions and Discussion } & 59\end{array}$

$\begin{array}{ll}\text { References } & 62\end{array}$ 


\section{List of Tables}

3.1 A movie rating table . . . . . . . . . . . . . . . . . 13

3.2 Equivalence classes formed based on Table 3.1 . . . . . . . . . . . . 14

4.1 Payoff table for a two-player GTRS based game . . . . . . . . . . . . 21

4.2 Payoff table for the competitive game between accuracy and coverage 25

5.1 Five featured data samples on MovieLens . . . . . . . . . . . . . 34

6.1 Training results on Sample $_{1-50} \ldots \ldots \ldots$. . . . . . . . . . 39

6.2 Test results on Sample $_{1-50} \ldots \ldots$. . . . . . . . . . . . . . 39

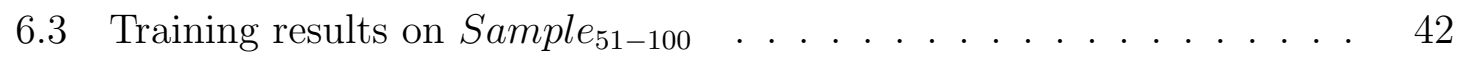

6.4 Test results on Sample $_{51-100} \ldots \ldots \ldots \ldots$. . . . . . . . . . 42

6.5 Training results on Sample $e_{101-150} \ldots \ldots$. . . . . . . . . . . 43

6.6 Test results on Sample $e_{101-150} \ldots \ldots \ldots$. . . . . . . . . . 43

6.7 Training results on Sample $e_{151-200} \ldots \ldots \ldots \ldots$. . . . . . . . 44

6.8 Test results on Sample $e_{151-200} \ldots \ldots \ldots$. . . . . . . . . . 44

6.9 Training results on Sample $e_{201-250} \ldots \ldots \ldots \ldots$. . . . . . . . 45

6.10 Test results on Sample $_{201-250} \ldots \ldots \ldots$. . . . . . . . . 45

6.11 Accuracy of the two prediction algorithms on the featured data samples 46

6.12 Coverage of the two prediction algorithms on the featured data samples 46

6.13 Categorization of recommendation decisions . . . . . . . . . . 50 
6.14 Precision and recall of the two prediction algorithms on the featured data samples . . . . . . . . . . . . . . . .

6.15 TPR and FPR of the two prediction algorithms on the featured data

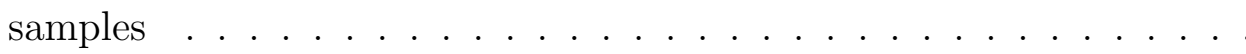




\section{List of Figures}

5.1 User distribution of the featured samples . . . . . . . . . . 35

6.1 Accuracy of the two prediction algorithms on the featured data samples 46

6.2 Coverage of the two prediction algorithms on the featured data samples 46 


\section{Chapter 1}

\section{Introduction}

Recommender systems predict a user's preference among a collection of items by aggregating and analyzing suggestions from similar users [3]. Through the use of data mining techniques, recommender systems help users to find items that they are interested in without searching through the enormous amount of information on the internet [4].

Different approaches are involved in the design phase of a recommender system [38]. Collaborative filtering ( $\mathrm{CF}$ ) predicts a user's opinion on an item by combining similar users' opinions on this specific item [42]. CF is the most commonly used recommendation technique to date as it makes moderately accurate recommendations and takes less effort to implement [23]. However, traditional CF algorithms rely highly on the rating density of the database [1], as the task of finding similarity among different users gets harder when fewer rating records are around [43].

One effective solution to this problem is to involve model-based methods in the implementation phase of a CF recommender system [41]. Through the use of data 
mining techniques, model-based methods transfer the preference information in the database into a preference model [10]. The task of making recommendations for new users no longer relies on the rating density of the database as the system will approach to the preference model instead of the original database. The Pawlak rough set [32] model is one of the effective techniques to implement model-based methods in $\mathrm{CF}$ recommender systems $[9,15,26]$.

The Pawlak rough set model is a powerful mathematical tool to deal with incomplete information. It forms equivalence classes with users that share similar interests on training data, and makes predictions with these formed equivalence classes on test data [6]. One limitation of the Pawlak rough set based recommendations is that as the model is intolerant to errors, its predictions are only applicable for a limited portion of users. However, this limitation could be eliminated through the use of the game-theoretic rough set (GTRS) model [47].

As a quantitative generalization of the Pawlak rough set model [37], the GTRS model helps the Pawlak rough set model with its error-intolerance and broadens its practical application. It formulates a competitive game between accuracy and coverage, the two most prominent metrics in recommender system evaluation. A threshold pair $\left(\alpha^{\prime}, \beta^{\prime}\right)$ that achieves a tradeoff between the two considered metrics will be returned once the competitive game is completed [7]. The GTRS threshold pair is then used to determine the three rough set regions and carry out rough set analysis.

As the rating pattern of the considered user group may have an impact on the performance of a CF recommendation algorithm [16], we run a comparative study between the Pawlak rough set model and the GTRS model using various data samples with different rating patterns. Both of the considered models are used to predict user preferences on five featured data samples formed by users with different number of rating records. However, when their recommendation performance are evaluated 
and compared with each other, the results provided by different evaluation metrics might be different [16]. Therefore, involving multiple evaluation metrics to test the performance of the two considered models helps us to make sure that a thorough comparative evaluation is achieved. In this thesis, besides accuracy and coverage, two decision support metrics are used to examine the classification ability of the two models: precision-recall, and receiver operating curve (ROC curve)-based metrics.

The remainder of the thesis consists of 6 different parts.

In Chapter 2, we give an overview of the related works that have been carried out in the field of recommender system research along with some of their limitations. Chapter 3 introduces some important concepts about the Pawlak rough set model and how it is used to make personalized recommendations. Chapter 4 gives an insight of how the GTRS model is used to formulate a competitive game between accuracy and coverage, which two of the metrics that are commonly used in recommender system evaluation. This chapter also indicates how the GTRS threshold pair is determined and how it could possibly improve the quality of the Pawlak rough set based recommendations.

In Chapter 5, some data preprocessing and partitioning are performed on the original dataset to form user groups with selected users that have a certain range of rating records. In Chapter 6, the Pawlak rough set model and the GTRS model are used to predict user preferences on the featured user groups formed in the last section respectively. Performance evaluations are carried out to compare the recommendation quality of the two models with each other, as well as to address the problem of how their performance vary on user groups with different rating patterns. Finally a summary, a conclusion, and limitations of our approach are discussed in Chapter 7. 


\section{Chapter 2}

\section{Background Knowledge and Related Work}

Different approaches are involved to make personalized recommendations in the design phase of a recommender system [2]. Collaborative filtering, content based filtering, knowledge based filtering and demographic based filtering are by far the most commonly used approaches to predict user preferences in the field of recommender system research [10].

Collaborative filtering predicts a user's preference by aggregating preference information from users that are considered to be similar with the target user [42]. Content based filtering analyzes a set of related documents to set up a profile for each target user and make predictions based on the inferred user profile [31]. Knowledge based filtering studies the data that are related to the target user's needs to make preference predictions [13]. Demographic based filtering divides all users in the dataset into demographic user groups with regard to their personal attributes and makes recommendations for each target user based on the demographic group that he or she belongs to [34]. The information sources that these four approaches use to generate 
recommendations is what make them different from each other. Collaborative filtering makes use of a user's previous rating history to discover similarities among different users, while content-based filtering infers a user profile with the content of the related documents. Knowledge based filtering takes the need of each target user as an input, while demographic filtering analyzes users' personal attributes to form demographic user groups. Among all of the approaches, collaborative filtering is by far the most popular technique as it takes less effort to implement and makes moderately accurate recommendations [25].

The methods involved in the implementation phase a $\mathrm{CF}$ recommender system could be further divided into two categories: memory-based methods and modelbased methods [10]. Memory-based methods maintain a database to store rating information of all users and make calculations across the whole database whenever a prediction needs to be made. These methods treat the entire database as a user group that has similar interests and temporarily identify a list of neighbours for each target user. These neighbours are a group of users that share common interests with the target user. Recommendations for the target user are generated by combining the opinions from these neighbours. Memory-based methods are widely implemented in e-commerce websites as they are comparatively easy to implement and moderately efficient in practical application [43]. However, the performance of memory-based methods is affected by the rating density of the database as finding users with similar interest gets harder when fewer users and rating records could be found [23].

On the other hand, model-based methods transfer the existing information in the database into a user preference model through the use of data mining algorithms [36]. In the model construction process, the system is able to recognize the unique pattern from training data and make suitable recommendations on test data [10]. When a new user's information is input into the system, the system will approach to the 
preference model instead of the original database to generate personalized recommendations. Therefore, the performance of model-based algorithms are less likely to be affected by the rating density of the database [31]. It is believed that by using training data to construct a preference model beforehand and making recommendations with the constructed preference model [36], model-based algorithms are able to overcome the limitation of memory-based algorithms.

Different data mining models are used to predict user preferences in modelbased CF recommender systems [40]. Some of the well-known models that are commonly used in model-based CF recommender systems include the Bayesian belief nets model [12], the clustering model [46], the latent semantic CF model [4], and the Pawlak rough set model [31]. The Bayesian belief nets model assigns a decision tree for each node in the Bayesian network. In each decision tree, a node represents a considered item and the state of the node indicates the possible rating of the considered item [29]. The clustering model divides the original dataset into different clusters and make predictions for a user according to the cluster he or she belongs to [14]. The latent semantic model involves latent class variables to form user communities and make recommendations by calculating the overlap among these communities [44]. The Pawlak rough set model discovers similarity among different users through the concept of equivalence class and predicts a new user's opinion based on the equivalence class he or she belongs to [24].

However, the Pawlak rough set based recommendations are only applicable for a limited portion of users due to the error-intolerant nature of the Pawlak rough set model. The GTRS model improves the quality of the Pawlak rough set based recommendations by determining the three rough set regions with a threshold pair $(\alpha, \beta)$. The GTRS threshold pair is obtained by formulating a competitive game between 
two of the most prominent recommendation evaluation metrics, accuracy and coverage [6]. When a tradeoff between the two evaluation metrics is achieved, the solution to the competitive game, i.e., an threshold pair $\left(\alpha^{\prime}, \beta^{\prime}\right)$ will be returned [7]. The three rough set regions are determined using threshold pair $\left(\alpha^{\prime}, \beta^{\prime}\right)$ and the predictions are generated based on the three determined rough set regions. Details about how the threshold pair $\left(\alpha^{\prime}, \beta^{\prime}\right)$ is computed and why the incorporation of the this threshold pair is able to make the Pawlak rough set based recommendations applicable for more users will be given in Chapter 4 [6].

On the other hand, the performance of a CF recommendation algorithm may be affected by the rating pattern of the considered user group [16]. The rating pattern of the considered user group could be represented by the number of rating records each user has in the given dataset which directly affects the difficulty of finding users that are similar with the target user. In Chapter 6, comparative experiments are carried out to address the problem of how the performance of the Pawlak rough set based and the GTRS based recommendations vary on user groups with different rating patterns.

Different evaluation metrics are introduced to evaluate the performance of a recommender system. The metric of accuracy and the metric of coverage are two of the most popular ones among all of them [40]. Accuracy computes how close the recommender system's predictions are to the actual preference of the target user [23]. Coverage measures the portion of users for whom recommendations could be given using only the prediction algorithm [23]. However, when the performance of two recommendation algorithms are evaluated and compared with each other, different evaluation metrics may provide different results [16]. An algorithm that is winning in one aspect might be losing in the other, since each evaluation metric reflects a certain performance aspect of the considered recommendation algorithm. Therefore, it is important that we involve multiple evaluation metrics to compare the performance 
of the two considered models.

Aside from accuracy and coverage, the classification ability is also an important performance aspect for a recommendation algorithm. The classification ability of a recommendation algorithm is represented by the ratio of correct and incorrect decisions on the considered items and could be computed through the calculation of decision-support metrics [23]. The decision on an item is considered correct if it is (a) both recommended and preferred by the target user, or (b) not recommended to the target user and is also an item that the target user does not prefer. Otherwise, the decision on the considered item is incorrect.

In this thesis, two decision support metrics, precision-recall and ROC curve-based metrics are used to examine the classification ability of the two considered models [11]. Precision measures the probability that a recommended item is preferred by the target user, while recall describes the probability that a preferred item is recommended to the considered user [23]. The ROC curve-based metrics calculate the true positive rate (TPR), which computes the ratio of recommended and preferred items to all the items in the preferred item set, and false positive rate ( $F P R)$, which measures the ratio of recommended and not preferred items to all the items in the not preferred item set. 


\section{Chapter 3}

\section{Pawlak Rough Set Based \\ Recommendations}

\subsection{The Pawlak Rough Set Model}

The Pawlak rough set model approximates a set $C$ by a pair of lower and upper

approximations [6]. Let $U$ be a set called universe, and let $[x]$ be an equivalence class that is formed based on an equivalence relation on $U$ [33]. Set $C$ that is being approximated is normally a subset of set $U$. The lower and upper approximation for set $C$ are defined as follows [32],

$$
\begin{gathered}
\underline{\operatorname{apr}}(C)=\{x \in U \mid[x] \subseteq C\} \\
\overline{\operatorname{apr}}(C)=\{x \in U \mid[x] \cap C \neq \emptyset\}
\end{gathered}
$$


With the definitions of the upper and lower approximations of set $C$, the three rough set regions, the positive, the negative and the boundary region are calculated as follows,

$$
\begin{gathered}
\operatorname{POS}(C)=\underline{\operatorname{apr}}(C), \\
N E G(C)=\overline{\operatorname{apr}}(C)^{c} \\
B N D(C)=\overline{\operatorname{apr}}(C)-\underline{\operatorname{apr}(C)}
\end{gathered}
$$

\subsection{The Probabilistic Rough Set Model}

The probabilistic rough set model calculates the conditional probability of each equivalence class, which is defined as the degree of overlap between the equivalence class $[x]$ and the set $C$. In the probabilistic rough set model, the conditional probability of equivalence class $[x]$ is calculated using the following equation,

$$
P(C \mid[x])=\frac{|C \cap[x]|}{|[x]|}
$$

Aside from the conditional probabilities of the equivalence classes on $U$, a threshold pair $(\alpha, \beta)$ is also introduced to determine the upper and the lower approximations of set $C$. All the equivalence classes on $U$ with their conditional probabilities satisfying the condition $P(C \mid[x]) \geq \alpha$ form a lower approximation of set $C$. Likewise, all the equivalence classes on $U$ with their conditional probabilities satisfying the condition $P(C \mid[x])>\beta$ form an upper approximation of set $C$. For instance, if $(\alpha, \beta)=(0.5,0.5)$, an equivalence class $[x]$ with a conditional probability 
of $P(C \mid[x])=0.6$ will be classified as part of the upper approximation of set $C$ as its conditional probability satisfies the condition of $P(C \mid[x]) \geq \alpha$. On the other hand, if $(\alpha, \beta)=(0.8,0.2)$, then the same equivalence class $[x]$ will be classified as part of the lower approximation of set $C$ as its conditional probability satisfies the condition of $P(C \mid[x])>\beta$.

With the conditional probability of each equivalence class calculated and the threshold pair $(\alpha, \beta)$ determined, the upper approximation and the lower approximation of set $C$ are defined as follows,

$$
\begin{aligned}
& \underline{\operatorname{apr}}_{(\alpha, \beta)}(C)=\{x \in U \mid P(C \mid[x]) \geq \alpha\} \\
& \overline{\operatorname{apr}}_{(\alpha, \beta)}(C)=\{x \in U \mid P(C \mid[x])>\beta\}
\end{aligned}
$$

Similar to the Pawlak rough set model, with the definition of the upper approximation and the lower approximation of set $C$, the three probabilistic rough set regions are determined as follows,

$$
\begin{gathered}
\operatorname{POS}_{(\alpha, \beta)}(C)=\underline{\operatorname{apr}}(\alpha, \beta) \\
N E G_{(\alpha, \beta)}(C)=\left\{\overline{a p r}_{(\alpha, \beta)}(C)\right)^{c}=\{x \in U \mid P(C \mid[x]) \leq \beta\} \\
B N D_{(\alpha, \beta)}(C)=\overline{a p r}_{(\alpha, \beta)}(C)-\underline{\operatorname{apr}}(C)=\{x \in U \mid \beta<P(C \mid[x])<\alpha\}
\end{gathered}
$$

The probabilistic rough set model eliminates the limitation of the Pawlak rough set model by introducing the concept of conditional probability and a pair of thresholds $(\alpha, \beta)$. With the conditional probability of each equivalence class calculated and a valid threshold pair $(\alpha, \beta)$ determined, we are able to define the three rough set regions and carry out rough set analysis. An example of how the Pawlak rough set model 
and the probabilisitic rough set model could be used to predict user preferences will be given in the next section.

\subsection{Pawlak Rough Set Based Recommendations}

Table 3.1 is an example of a movie rating table constructed using the rating information in the MovieLens dataset. For instance, let us consider a user set $U$ with a total of 16 users $U_{1}, U_{2}, \ldots, U_{16}$, i.e., $U=\left\{U_{1}, U_{2}, \ldots, U_{16}\right\}$. The considered movie set $M=\{$ Movie 1, Movie $2, \ldots$, Movie 5$\}$ are made up with five different movies that have been rated by all the users in $U$.

Each cell in Table 3.1 describes a rating record made by a specific user with regard to a specific movie. For instance, the first cell in the first row represents a rating record made by user $U_{1}$ with regard to Movie1. For each user, a positive rating to a movie is considered to be a 'like' and is transferred into a ' + ' in the rating table. A negative rating to a movie is considered to be a 'dislike' and is transferred into a '-' in the rating table.

The goal of the rough set analysis is to make preference predictions on Movie5. In order to predict a user's opinion on Movie5, we combine the ratings that have been previously made on Movie5 and the target user's ratings on Movie1 - Movie4. As we are assuming users with the same preferences on Movie1 - Movie4 might share a similar taste in movies, users that are similar to the target user are discovered by analyzing the previous rating information on these four movies. The target user's opnion on Movie5 is predicted using similar users' rating information on Movie5.

In the rough set model, Movie1 - Movie4 are defined as the conditional attributes, 
Table 3.1: A movie rating table

\begin{tabular}{cccccc}
\hline & Movie1 & Movie2 & Movie3 & Movie4 & Movie5 \\
\hline$U_{1}$ & - & + & + & + & - \\
\hline$U_{2}$ & + & + & - & + & + \\
\hline$U_{3}$ & + & - & - & - & + \\
\hline$U_{4}$ & - & + & + & + & - \\
\hline$U_{5}$ & + & - & - & - & - \\
\hline$U_{6}$ & + & - & + & - & + \\
\hline$U_{7}$ & - & + & + & + & + \\
\hline$U_{8}$ & + & - & + & - & - \\
\hline$U_{9}$ & - & - & + & - & + \\
\hline$U_{10}$ & - & - & + & + & - \\
\hline$U_{11}$ & - & - & + & + & + \\
\hline$U_{12}$ & - & - & - & - & - \\
\hline$U_{13}$ & - & - & + & - & + \\
\hline$U_{14}$ & - & - & + & + & + \\
\hline$U_{15}$ & + & + & + & + & + \\
\hline$U_{16}$ & - & - & - & - & - \\
\hline
\end{tabular}

while Movie5 is defined as the decision attribute. In order to predict the value of the decision attribute, we need to first identify the users that share similar interests with the target user based on their ratings on the conditional attributes. The Pawlak rough set model identifies the similarities among different users by classifying users with the same conditional attribute values into the same equivalence class. An equivalence class $X_{i}$ is formed by a set of users with the same rating preferences on the conditional attribute movies, i.e., Movie1 - Movie4. For instance, user $U_{1}$ and $U_{4}$ both have a negative rating on Movie1 and positive ratings on Movie2 - Movie4. Therefore, they are considered to be similar with each other and are categorized into the same equivalence class $X_{1}$.

The users in the user set $U$ are classified into eight different equivalence classes according to the rating records they previously made on Movie1 - Movie4. The eight equivalence classes $X_{1}, X_{2}, \ldots, X_{8}$ formed based on Table 3.1 are described in Table 3.2.

When new users enter the system, we first identify the equivalence class they 
Table 3.2: Equivalence classes formed based on Table 3.1

\begin{tabular}{cc}
\hline$X_{1}=\left\{U_{1}, U_{4}\right\}$ & $X_{2}=\left\{U_{2}\right\}$ \\
$X_{3}=\left\{U_{3}, U_{5}, U_{7}\right\}$ & $X_{4}=\left\{U_{6}, U_{8}\right\}$ \\
$X_{5}=\left\{U_{9}, U_{13}\right\}$ & $X_{6}=\left\{U_{10}, U_{11}, U_{14}\right\}$ \\
$X_{7}=\left\{U_{12}, U_{16}\right\}$ & $X_{8}=\left\{U_{15}\right\}$ \\
\hline
\end{tabular}

belong to based on the rating records they previously made on Movie1 - Movie4. Then we predict their preferences on Movie5 according to the preferences of the equivalence classes they belong to, as we assume that users that share the same opinions on Movie1 - Movie4 might have similar tastes in movies. However, the preference prediction of the target user could not be specified if the users in the equivalence class do not agree with each other with regard to their opinions on Movie5. For instance, we are unable to tell whether equivalence class $X_{4}$ likes Movie5 or not as one of the users in the equivalence class likes the movie while the other does not. In the Pawlak rough set model, these equivalence classes are classified into the boundary region, which means the preferences of the users that belong to these equivalence classes could not be predicted. In other words, user preferences could only be predicted for the equivalence classes that belongs to the positive or negative region, which means recommendations are only applicable for a limited portion of users.

Although leaving out the equivalence classes in the boundary region reduces the possibility of making incorrect recommendations, only being able to make predictions for a limited portion of users is a drawback in its practical application. A solution to make the Pawlak rough set based recommendations applicable for more users is to introduce the concept of conditional probability in the probabilistic rough set model. The conditional probability of equivalence class $X_{i}$ is calculated using the following equation,

$$
P\left(C \mid X_{i}\right)=P\left(\text { Movie } 5=+\mid X_{i}\right)=\frac{\mid \text { Movie } 5=+\cap X_{i} \mid}{\left|X_{i}\right|}
$$


The conditional probabilities of the equivalence classes $X_{1}, \ldots, X_{8}$ in Table 3.2 are calculated as $0.0,1.0,0.7,0.5,1.0,0.7,0.0$, and 1.0 , respectively. These conditional probabilities represent the percentage of users that have previously made a positive rating on Movie5 in each equivalence class. According to the Pawlak rough set model, equivalence classes $X_{1}$ and $X_{7}$ with their conditional probabilities being $P\left(C \mid X_{1}\right)=0.0, P\left(C \mid X_{7}\right)=0.0$ belong to the positive region. Equivalence classes $X_{2}, X_{5}$, and $X_{8}$ with their conditional probabilities being $P\left(C \mid X_{2}\right)=1.0$, $P\left(C \mid X_{5}\right)=1.0$, and $P\left(C \mid X_{8}\right)=1.0$ belong to the negative region. However, the decision on which region the other three equivalence classes belong to could not be made with only the calculation of the conditional probabilities.

As discussed in the last section, in the probabilistic rough set model, aside from the conditional probabilities of each equivalence class, a pair of thresholds $(\alpha, \beta)$ is also needed to determine the three rough set regions. After calculating the conditional probabilities for all the equivalence classes, we need to compare these conditional probabilities with the threshold pair $(\alpha, \beta)$ to determine which region the equivalence classes belong to. An equivalence class with a conditional probability greater than threshold $\alpha$ will be classified into the positive region, and an equivalence class with a conditional probability less than $\beta$ will be classified into the negative region. Otherwise, the equivalence class stays in the boundary region. 


\subsection{Accuracy and Coverage of the Pawlak Rough Set Based Recommendations}

Different metrics have been proposed to evaluate the performance of recommendation algorithms, and accuracy and coverage are two of the most popular ones among all of them [40].

Assuming that examining the actual user preferences on the considered item set is possible, accuracy empirically computes how close the recommender system's predictions are to the actual user preferences [17]. Coverage measures the domain of users for whom recommendations could be generated using only the considered prediction algorithm [39]. These two evaluation metrics provide us different yet complimentary performance aspects of a recommendation algorithm [23].

In the rough set model, the metric of accuracy is defined as the ratio of the number of correctly classified objects in the positive region and the negative region to the total number of objects in these two regions. The metric of coverage is defined as the ratio of the total number of objects in the positive region and the negative region to the total number of objects in the universal set. If the threshold pair that is used to determine the three rough set regions is configured as $(\alpha, \beta)$, the metrics of accuracy and coverage are calculated using the following equations,

$$
\begin{gathered}
\operatorname{Accuracy}_{(\alpha, \beta)}=\frac{\left|\left(\operatorname{POS}_{\alpha, \beta}(C) \cap C\right) \cup\left(N E G_{\alpha, \beta}(C) \cap C^{c}\right)\right|}{\left|P O S_{\alpha, \beta}(C) \cup N E G_{\alpha, \beta}(C)\right|} \\
\operatorname{Coverage}_{(\alpha, \beta)}=\frac{\left|\operatorname{POS}_{\alpha, \beta}(C) \cup N E G_{\alpha, \beta}(C)\right|}{|U|}
\end{gathered}
$$

Both accuracy and coverage are the properties we want to pursue in the Pawlak rough set based recommendations, and we may want to optimize them both at the 
same time. However, this may not be possible in many cases. As we could observe from Eq 3.14, given a universal set $U$, the denominator $|U|$ is fixed. Therefore, the only way to improve coverage level is to increase the molecular $\mid P O S_{\alpha, \beta}(C) \cup$ $N E G_{\alpha, \beta}(C) \mid$. However, the molecular in Eq 3.14 is the denominator in Eq 3.13, therefore, whenever coverage increases, accuracy will decrease. An attempt to increase the coverage level will make recommendations applicable for more users, but will also end up with more not preferred recommendations. Therefore, instead of increasing them both at the same time, we try to realize a tradeoff between the two considered attributes.

The problem yet to solve is to what degree is this tradeoff acceptable. As the Pawlak rough set based recommendations are only applicable for a limited portion of users, how much sacrifice in accuracy level is acceptable in order to improve coverage level requires more tradeoff analysis. In this thesis, we realize the tradeoff between the two attributes through the use of the GTRS model. 


\section{Chapter 4}

\section{GTRS Based Recommendations}

\subsection{The Game-theoretic Rough Set Model}

The GTRS model determines the threshold pair $(\alpha, \beta)$ by formulating a competitive game among a finite set of game players, which are normally represented by the criteria that we want to pursue in the rough set analysis [22]. It moves objects in the boundary region into either the positive or the negative region by adjusting the threshold pair values in the competitive game.

Let $(\alpha, \beta)$ be the threshold pair that is used to determine the three rough set regions, and let $\left(c_{1}, c_{2}\right)$ be two of the criteria that we want to pursue in the rough set analysis. When the threshold pair is configured as $(\alpha, \beta)$, the payoff of the criteria $c_{1}$ and $c_{2}$ are described by $c_{1}(\alpha, \beta)$ and $c_{2}(\alpha, \beta)$. Since both $c_{1}$ and $c_{2}$ are the criteria we 
want to pursue, the goal of the rough set analysis is to select a threshold pair that could maximize the payoff of the two criteria at the same time, which is described as,

$$
\begin{gathered}
\operatorname{argmax}_{(\alpha, \beta)} c_{1}(\alpha, \beta), \\
\operatorname{argmax}_{(\alpha, \beta)} c_{2}(\alpha, \beta)
\end{gathered}
$$

However, as much as we want to optimize both $c_{1}(\alpha, \beta)$ and $c_{2}(\alpha, \beta)$ simultaneously, this might not be possible in real world scenario. The configuration of the threshold pair $(\alpha, \beta)$ has impacts on both criteria $c_{1}$ and criteria $c_{2}$ at the same time, which means an effort to increase the payoff of one criteria might result in a decrement in another criteria.

The GTRS model provides a solution to this problem by formulating a competitive game to realize a tradeoff between or among the considered criteria. Formally, a game is defined as a tuple $\{P, S, u\}[27]$ where,

- $P=\left\{p_{1}, p_{2}, \ldots, p_{n}\right\}$ is a finite set of players;

- $S=S_{1} \times S_{2} \times \ldots \times S_{n}$, where $S_{i}$ is a finite set of strategies available for player $p_{i}$ to choose from. Each strategy in the strategy set $S_{i}$ represents a different level of adjustment in the threshold pair $(\alpha, \beta)$. A strategy profile $S=\left\{s_{1}, s_{2}, \ldots, s_{n}\right\}$ is a combination of strategies selected by players $\left\{p_{1}, p_{2}, \ldots, p_{n}\right\}$, where player $p_{i}$ plays strategy $s_{i}$;

- $u=\left\{u_{1}, u_{2}, \ldots, u_{n}\right\}$ is a set of payoff functions calculated with regard to each strategy profile. 
In a GTRS based game, the selection of players is solely determined by the criteria we want to pursue in the decision making process. The strategies are considered as changes in the criteria levels, which is achieved by modifying the threshold levels [5]. The goal of the game is to find a solution to the game, which is represented by a strategy profile $\left(s_{1}, s_{2}, \ldots, s_{n}\right)$.

The concept of Nash equilibrium is used to determine a game solution in a GTRS based competitive game. For instance, in the above discussed two-player game between criteria $c_{1}$ and criteria $c_{2}$, a strategy profile $\left(s_{i}, s_{j}\right)$ is considered Nash equilibrium if the following two conditions hold,

$$
\begin{aligned}
& \text { for all } k \neq i, u_{c_{1}}\left(s_{i}, s_{j}\right) \geq u_{c_{1}}\left(s_{k}, s_{j}\right) \\
& \text { for all } k \neq j, u_{c_{2}}\left(s_{i}, s_{j}\right) \geq u_{c_{2}}\left(s_{i}, s_{k}\right)
\end{aligned}
$$

The first condition indicates that player $c_{1}$ achieves the best payoff by playing strategy $s_{i}$, provided that player $c_{2}$ has his or her strategy fixed as $s_{j}$. Similarly, the second condition suggests player $c_{2}$ achieves the best payoff by playing strategy $s_{j}$ given that player $c_{1}$ has his or her strategy fixed as $s_{i}$. When both conditions are reached, neither of the players could achieve a better payoff by adjusting his or her own strategy selection. In other words, a balance point between the two players is realized when Nash Equilibrium is achieved. 
Table 4.1: Payoff table for a two-player GTRS based game

\begin{tabular}{|c|c|c|c|c|}
\hline & \multicolumn{3}{|c|}{$c_{2}$} \\
\hline & & $s_{1}$ & $s_{2}$ & $\ldots$ \\
\hline \multirow{3}{*}{$c_{1}$} & $s_{1}$ & $\begin{array}{l}\left\langle u_{C_{1}}\left(s_{1}, s_{1}\right),\right. \\
\left.u_{C_{2}}\left(s_{1}, s_{1}\right)\right\rangle\end{array}$ & $\begin{array}{l}\left\langle u_{C_{1}}\left(s_{1}, s_{2}\right),\right. \\
\left.u_{C_{2}}\left(s_{1}, s_{2}\right)\right\rangle\end{array}$ & $\begin{array}{l}\cdots \\
\cdots\end{array}$ \\
\hline & $s_{2}$ & $\begin{array}{l}\left\langle u_{C_{1}}\left(s_{2}, s_{1}\right),\right. \\
\left.u_{C_{2}}\left(s_{2}, s_{1}\right)\right\rangle\end{array}$ & $\begin{array}{l}\left\langle u_{C_{1}}\left(s_{2}, s_{2}\right),\right. \\
\left.u_{C_{2}}\left(s_{2}, s_{2}\right)\right\rangle\end{array}$ & $\begin{array}{l}\cdots \\
\cdots\end{array}$ \\
\hline & $\ldots$ & $\ldots$ & $\ldots$ & $\ldots$ \\
\hline
\end{tabular}

\subsection{Formulating a Two-player GTRS Based Game}

Let us look at a classic two-player GTRS based game formed between the above discussed criteria $c_{1}$ and $c_{2}$, the two criteria that we want to pursue in the rough set analysis. In the competitive game, both criteria $c_{1}$ and $c_{2}$ are considered as game players. Different kinds of adjustments of the threshold pair $(\alpha, \beta)$ are the game strategies that each player could choose from. Supposing that according to the two players' strategy selections, the threshold pair is configured as $(\alpha, \beta)$. The payoff of the two players $c_{1}$ and $c_{2}$ are then calculated respectively using the corresponding payoff functions $u_{c_{1}}(\alpha, \beta)$ and $u_{c_{2}}(\alpha, \beta)$.

In the payoff table, each cell represents the payoff of the two game players calculated with regard to the corresponding strategy profile. For instance, the top left cell contains $u_{C_{1}}\left(s_{1}, s_{1}\right)$ and $u_{C_{2}}\left(s_{1}, s_{1}\right)$ that is calculated according to strategy profile $\left(s_{1}, s_{1}\right)$. The strategy combination of the two players corresponding to the strategy profile is $c_{1}$ playing $s_{1}$ and $c_{2}$ playing $s_{2}$. With the payoff of each strategy profile calculated using the corresponding payoff functions, a payoff table like Table 4.1 will be formed.

The solution concept Nash equilibrium is determined by going through each cell with an exhaustive search method to find the best payoff in the payoff table. A 
threshold pair $\left(\alpha^{\prime}, \beta^{\prime}\right)$ which represents a solution to the competitive game is calculated using the Nash Equilibrium strategy profile. With this GTRS threshold pair, a tradeoff between the two criteria $c_{1}$ and $c_{2}$ is realized. In the next section, a two-player GTRS based competitive game is formulated to achieve a tradeoff between criteria accuracy and criteria coverage.

\subsection{Formulating a Competitive Game between Accuracy and Coverage}

As discussed in the last chapter, the role of the GTRS model is to realize a tradeoff between accuracy and coverage by formulating a competitive game between them. In order to formulate a GTRS based competitive game between accuracy and coverage, we need to first identify the three components of the game, i.e, the players, the strategies, and the payoff functions.

The players of the competitive game should be the properties for whom a tradeoff needs to be made. As we are considering a tradeoff between accuracy and coverage, these two attributes are selected as game players [6], i.e., $P=\{$ Accuracy, Coverage $\}$.

The strategies are sets of moves that the players could choose from. In the GTRS model, strategies are realized by making corresponding adjustments in the thresholds level. To better compare with the Pawlak rough set model, in a GTRS based competitive game, the threshold pair is initially configured as $(\alpha, \beta)=(1,0)$, where the accuracy level is at the highest and the coverage level is at the lowest. As a result, player accuracy and player coverage have three different types of strategies to choose from, which is to decrease $\alpha$, to increase $\beta$, or to decrease $\alpha$ and increase $\beta$ at the 
same time, i.e., $s_{1}=\alpha \downarrow, s_{2}=\beta \uparrow, s_{3}=\alpha \downarrow \beta \uparrow$. In one iteration of the game, player accuracy and player coverage each chooses one strategy from the strategy set $s_{1}, s_{2}, s_{3}$. When a player plays a strategy, corresponding adjustments are made to the threshold pair $(\alpha, \beta)$. For instance, when player accuracy chooses to play strategy $s_{3}$, an increment of 0.05 is made to threshold $\beta$, and a decrement of 0.05 is made to threshold $\alpha$. Therefore, based on the strategy selection of the two players, four types of threshold adjustments, $\alpha \downarrow, \alpha \downarrow \downarrow, \beta \uparrow, \beta \uparrow \uparrow$, could possibly be applied to threshold $\alpha$ and threshold $\beta$. Threshold adjustment $\alpha \downarrow$ describes the situation where one player suggests to decrease threshold $\alpha$. Similarly, threshold adjustments $\alpha \downarrow \downarrow, \beta \uparrow$, $\beta \uparrow \uparrow$ describe the scenarios where two players suggest to decrease threshold $\alpha$, one player suggests to increase threshold $\beta$, and two players suggest to increase threshold $\beta$, respectively.

$$
\begin{gathered}
\alpha \downarrow: \text { single player suggests to decrease } \alpha, \\
\alpha \downarrow \downarrow: \text { both the players suggest to decrease } \alpha, \\
\beta \uparrow: \text { single player suggests to increase } \beta, \\
\beta \uparrow \uparrow: \text { both the players suggest to increase } \beta .
\end{gathered}
$$

These four types of threshold level adjustments suggest how each strategy combination has a unique impact on the threshold pair. For instance, if the strategy combination of player accuracy and player coverage is $\left(s_{1}, s_{1}\right)$, which means both players choose to decrease $\alpha$, the threshold level adjustment is then determined as $(\alpha \downarrow \downarrow, \beta)$. Likely, if the strategy combination of the two players is $\left(s_{3}, s_{3}\right)$, which means both players choose to decrease $\alpha$ and increase $\beta$ at the same time, the threshold level adjustment is determined as $(\alpha \downarrow \downarrow, \beta \uparrow \uparrow)$. 
The payoff functions are used to measure the outcome of a player playing a specific strategy. Since accuracy and coverage levels are what the players want to pursue in the competitive game, the values of these two attributes are calculated as the payoffs of the two game players. An adjustment in thresholds levels directly affects the determination of the three rough set regions, which further influences the payoffs of the two game players. The definition of accuracy and coverage in Eq 3.13 and Eq 3.14 are used as payoff functions as we are calculating accuracy and coverage level according to the threshold pair values. For a particular strategy profile $\left(s_{m}, s_{n}\right)$ with its threshold pair values configured as $(\alpha, \beta)$, the payoff functions of the two players are described by,

$$
\begin{gathered}
u_{A}\left(s_{m}, s_{n}\right)=\operatorname{Accuracy}_{(\alpha, \beta)}=\frac{\left|\left(\operatorname{POS}_{\alpha, \beta}(C) \cap C\right) \cup\left(N E G_{\alpha, \beta}(C) \cap C^{c}\right)\right|}{\left|P O S_{\alpha, \beta}(C) \cup N E G_{\alpha, \beta}(C)\right|} \\
u_{C}\left(s_{m}, s_{n}\right)=\text { Coverage }_{(\alpha, \beta)}=\frac{\left|P O S_{\alpha, \beta}(C) \cup N E G_{\alpha, \beta}(C)\right|}{|U|}
\end{gathered}
$$

After one iteration of the competitive game, a payoff table like Table 4.2 will be formed. The rows in Table 4.2 represent the strategy selection of player accuracy while the columns describe the strategy selection of player coverage. Each cell is assigned with a set of payoffs calculated using the payoff functions with regard to the strategy selections of the two game players. The adjustments in threshold level $\alpha \downarrow, \downarrow \downarrow, \beta \uparrow, \beta \uparrow \uparrow$ for each strategy profile is determined using Eq 4.25 - Eq 4.28.

The solution to the competitive game (Nash equilibrium) is calculated by going through each cell in the payoff table to check if the conditions in Eq 4.17 and Eq. 4.18 hold. The strategy profile $\left(s_{i}, s_{j}\right)$ that yields the conditions of Nash equilibrium is considered to be the solution to the competitive game. The threshold pair $\left(\alpha^{\prime}, \beta^{\prime}\right)$ 
Table 4.2: Payoff table for the competitive game between accuracy and coverage

\begin{tabular}{|c|c|c|c|c|}
\hline \multicolumn{2}{|c|}{} & \multicolumn{3}{c|}{ Coverage } \\
\cline { 3 - 5 } & $s_{1}=\alpha \downarrow$ & $s_{2}=\beta \uparrow$ & $s_{3}=\alpha \downarrow \beta \uparrow$ \\
\hline \multirow{5}{*}{ Accuracy } & $s_{1}=\alpha \downarrow$ & $\left\langle u_{A}(\alpha \downarrow \downarrow, \beta)\right.$, & $\left\langle u_{A}(\alpha \downarrow, \beta \uparrow)\right.$, & $\left\langle u_{A}(\alpha \downarrow \downarrow, \beta \uparrow)\right.$, \\
& & $\left.u_{C}(\alpha \downarrow \downarrow, \beta)\right\rangle$ & $\left.u_{C}(\alpha \downarrow, \beta \uparrow)\right\rangle$ & $\left.u_{C}(\alpha \downarrow, \beta \uparrow)\right\rangle$ \\
\cline { 2 - 5 } & $s_{2}=\beta \uparrow$ & $\left\langle u_{A}(\alpha \downarrow, \beta \uparrow)\right.$, & $\left\langle u_{A}(\alpha, \beta \uparrow \uparrow)\right.$, & $\left\langle u_{A}(\alpha \downarrow, \beta \uparrow \uparrow)\right.$, \\
& & $\left.u_{C}(\alpha \downarrow, \beta \uparrow)\right\rangle$ & $\left.u_{C}(\alpha, \beta \uparrow \uparrow)\right\rangle$ & $\left.u_{C}(\alpha \downarrow, \beta \uparrow \uparrow)\right\rangle$ \\
\cline { 2 - 5 } & $s_{3}=\alpha \downarrow \beta \uparrow$ & $\left\langle u_{A}(\alpha \downarrow \downarrow, \beta \uparrow)\right.$, & $\left\langle u_{A}(\alpha \downarrow, \beta \uparrow \uparrow)\right.$, & $\left\langle u_{A}(\alpha \downarrow \downarrow, \beta \uparrow \uparrow)\right.$, \\
& & $\left.u_{C}(\alpha \downarrow \downarrow, \beta \uparrow)\right\rangle$ & $\left.u_{C}(\alpha \downarrow, \beta \uparrow \uparrow)\right\rangle$ & $\left.u_{C}(\alpha \downarrow \downarrow, \beta \uparrow \uparrow)\right\rangle$ \\
\hline
\end{tabular}

calculated according to the solution strategy profile $\left(s_{i}, s_{j}\right)$ is then used to obtain the three rough set regions. A balanced accuracy and coverage level could be achieved by determining the rough set regions with the threshold pair attained in the GTRS based competitive game.

\subsection{Determining GTRS Thresholds with a Tradeoff between Accuracy and Coverage}

A learning mechanism will be formed by making the competitive game iterative and constantly adjusting the value of the four threshold level adjustment variables, i.e., $\alpha \downarrow, \alpha \downarrow \downarrow, \beta \uparrow, \beta \uparrow \uparrow$. The learning process will make the threshold level adjustments more effective after every iteration of the competitive game. An algorithmic realization of the iterative game is described in Algorithm 1.

To better compare with the Pawlak rough set model, the threshold pair $(\alpha, \beta)$ that is used to determine the three rough set regions is initially configured as $(\alpha, \beta)=(1,0)$ which is set according to the definition in the Pawlak rough set model. After one iteration of the game, the game solution is determined by going through the payoff table to check if the conditions of Nash Equilibrium hold. The corresponding threshold 


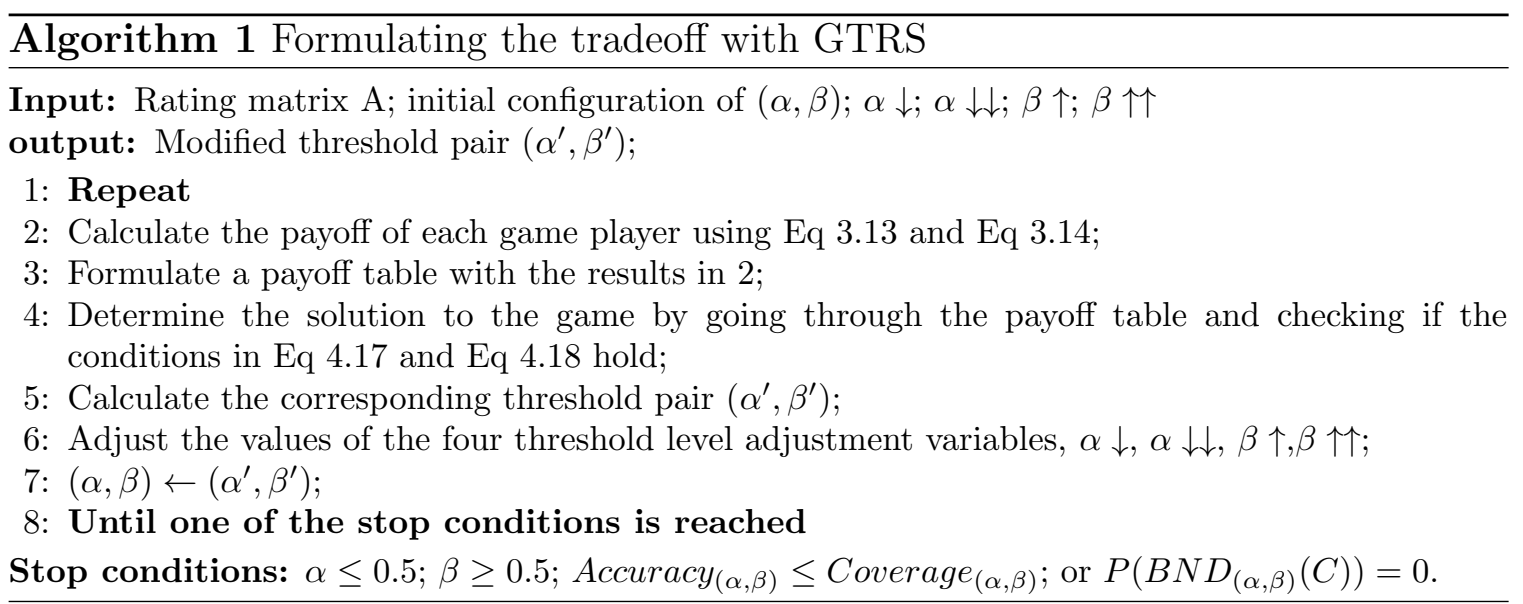

pair $\left(\alpha^{\prime}, \beta^{\prime}\right)$ is used as the initial threshold pair configuration in the next iteration of the game.

Besides the initial configuration of the threshold pair, the four threshold level adjustment variables $\alpha \downarrow, \alpha \downarrow \downarrow, \beta \uparrow, \beta \uparrow \uparrow$ also change after one iteration of the game is completed. The values of the adjustment variables are mainly determined by the improvement in coverage level in the last iteration of the game, while the constant $c$ is introduced to manage the level of change in these variables. The constant $c$ is initially configured as 2 and could be reconfigured at any value greater than 1 according to the desired level of change in the thresholds adjustment variables. Whenever one iteration of the game is completed, the four threshold level adjustment variables are calculated once again using the following equations [6],

$$
\begin{gathered}
\alpha \downarrow=\alpha-\left(\alpha \times\left(\text { Coverage }\left(\alpha^{\prime}, \beta^{\prime}\right)-\operatorname{Coverage}(\alpha, \beta)\right)\right) \\
\alpha \downarrow \downarrow=\alpha-c\left(\alpha \times\left(\text { Coverage }\left(\alpha^{\prime}, \beta^{\prime}\right)-\operatorname{Coverage}(\alpha, \beta)\right)\right) \\
\beta \uparrow=\beta-\left(\beta \times\left(\operatorname{Coverage}\left(\alpha^{\prime}, \beta^{\prime}\right)-\operatorname{Coverage}(\alpha, \beta)\right)\right) \\
\beta \uparrow \uparrow=\beta-c\left(\beta \times\left(\operatorname{Coverage}\left(\alpha^{\prime}, \beta^{\prime}\right)-\operatorname{Coverage}(\alpha, \beta)\right)\right)
\end{gathered}
$$


By changing variables $\alpha \downarrow, \alpha \downarrow \downarrow, \beta \uparrow, \beta \uparrow \uparrow$ after every iteration of the game, the adjustments in threshold levels will become more and more effective in terms of realizing the tradeoff between accuracy and coverage. The GTRS based competitive game adjusts the threshold levels and moves items in the boundary region to either the positive or the negative region. Therefore, the iteration will stop whenever the boundary region is empty. Moreover, since the goal of the game is to increase the coverage level with minimum sacrifice in accuracy level, the iterative process will also stop if the payoff of coverage exceeds the payoff of accuracy. Likewise, there are two more stop conditions. If $\alpha<0.5$ or if $\beta \geq 0.5$, the iteration will stop to prevent the attempts to increase coverage level from causing unacceptable decrement in accuracy level. These stop conditions can be mathematically expressed as,

$$
\begin{gathered}
\alpha<0.5 \\
\beta \geq 0.5 \\
\operatorname{Accuracy}(\alpha, \beta) \leq \text { Coverage }(\alpha, \beta) \\
P\left(B N D_{(\alpha, \beta)}(C)\right)=0
\end{gathered}
$$

We conduct the competitive game on training data and calculate the Nash Equilibrium strategy pair in each iteration of the game. Then we modify the threshold pair $(\alpha, \beta)$ using the Nash Equilibrium strategy pair and set the modified threshold pair $\left(\alpha^{\prime}, \beta^{\prime}\right)$ as the initial threshold pair, i.e., $(\alpha, \beta)=\left(\alpha^{\prime}, \beta^{\prime}\right)$. We keep adjusting the threshold levels until one of the stop conditions is reached and the threshold pair $\left(\alpha^{\prime}, \beta^{\prime}\right)$ attained in the last iteration of the game is used to classify objects on test data. Approximating test data with the GTRS threshold pair $\left(\alpha^{\prime}, \beta^{\prime}\right)$ will not only make the Pawlak rough set based recommendations applicable for more users, but 
also improve the recommendation performance in various ways.

However, the performance of a recommendation algorithm is also influenced by the number of rating records each user had in the considered data sample. The problem of how the performance of the Pawlak rough set based and the GTRS based recommendations vary on user groups with different rating patterns has yet to be addressed. Therefore, in Chapter 6, five sets of comparative experiments are carried out to compare the performance of the two considered models on data samples with different rating patterns. To form these data samples, some data preprocessing and partitioning are carried out on the original dataset. The details about the data preprocessing and partitioning process is described in the next chapter. 


\section{Chapter 5}

\section{Data Partitioning and Preprocessing}

Given a dataset, there are three important observations about CF recommendation algorithms [16],

- The performance of an algorithm is affected by the rating pattern of the user group, which could be represented by the number of rating records each user had in the considered data sample;

- The task of discovering the similarities among different users is always easier with the ratings on the frequently rated items compared to the infrequently rated ones;

- When the performance of two recommendation algorithms are compared with each other, the results provided by different evaluation metrics might be different. 
In order to address the above listed observations, some data preprocessing and data partitioning are conducted on the original dataset following the corresponding strategies,

- We classify all the users into smaller user groups by counting the number of rating records each user had in the considered dataset. The rating records in the original dataset is then partitioned into featured data samples according to the formed user groups;

- For each user group, we consider the frequently rated items to form equivalence classes, i.e., to discover similarities among different users;

- Aside from accuracy and coverage, we involve two decision support metrics, precision-recall, and the ROC curve-based metrics to evaluate the classification ability of the two models.

The first strategy considers the fact that the rating pattern of the considered user group has an impact on the performance of a CF recommendation algorithm. The number of rating records directly affects the difficulty of finding users that are similar with the target user. Besides, user groups with more rating records and user groups with fewer rating records have different rating behaviours. A user who tends to make more rating records are more likely to rate items positively, and a user who tends to make fewer rating records are more likely to rate items negatively [28]. Therefore, to make sure a thorough evaluation is achieved, we form featured user groups with different rating patterns. For each user, we count the number of rating records he or she had in the dataset, and then form user groups with the users that made a certain range of rating records. Featured data samples are formed by partitioning the rating 
records in the original dataset according to the formed user groups. For instance, let us consider a dataset $S$ with a total of four users, i.e, $U=\left\{U_{1}, U_{2}, U_{3}, U_{4}\right\}$. First, we count the number of rating records each user had in dataset $S$. Assuming that these four users have $5,20,10,15$ rating records in $S$ respectively, then $U_{1}$ and $U_{3}$ could be classified into user group $U_{1-10}$ with their rating records in the range of 1-10. Similarly, $U_{2}$ and $U_{4}$ could be classified into user group $U_{11-20}$ with the number of their rating records in the range of 11-20. The rating records in dataset $S$ is then partitioned according to the two formed user groups $U_{1-10}$ and $U_{11-20}$ to formulate two featured data samples, Sample St-10 $_{10}$ and Sample S1-20. $_{11}$.

The second strategy considers the fact that it is easier for any recommendation algorithm to discover similarities among different users with the ratings on frequently rated items. CF recommendation algorithms predict a user's opinion on one item by combining similar users' opinions on this specific item. In the rough set model, the similarities among different users are denoted by the concept of equivalence class. We consider users with the same opinions on all the items in the considered item set to be in the same equivalence class. With the ratings on frequently rated items, as all the considered items have been previously rated by lots of users in the dataset, the task of finding users with similar opinions on all the items in the considered item set is comparatively easy. The equivalence classes formed using the ratings on frequently rated items are also generally larger than the ones formed using the infrequently rated ones, as more similar users could be found for each target user. Therefore, to make sure that there are enough similar users in each equivalence class for a new user to learn from, we form equivalence classes using the ratings on the frequently rated items.

The third strategy considers the fact that the evaluation results provided by different evaluation metrics might be different, as each evaluation metric reflects a certain 
performance aspect of the considered recommendation algorithm. Beside accuracy and coverage, the classification ability is also important in the performance evaluation of a recommendation algorithm. The classification ability of a recommendation algorithm represents the ratio of correct and incorrect recommendation decisions, and could be measured through the use of decision-support metrics. Two decision support metrics, precision-recall, and ROC curve-based metrics are used to compare the classification ability of the two considered models. Precision computes the probability that a recommended item is preferred, and recall defines the ratio of prefered and recommended item to all the items in the preferred item set. The ROC curve-based metrics measures the ratio of recommended and preferred items to all the items in the preferred item set, and the ratio of recommended and not preferred items to all the items in the not preferred item set.

Performing data preprocessing and partitioning on the original dataset following the above strategies will lead to a thorough comparative evaluation. To implement these strategies, we first classify the users in the original dataset into user groups by counting the number of rating records they had in the dataset. Then all the ratings in the original dataset is partitioned into featured data samples according to the formed user groups. After these featured data samples are formed, we transfer the 5-star scale ratings into binary scale ratings. For each user group, the ratings for ten of the most frequently rated movies are selected to form equivalence classes, i.e., to discover similarities among different users. After the above data preprocessing process is completed, for each data sample, $80 \%$ of the rating information is used as training data, and the remaining $20 \%$ is used as test data.

The details about the data partitioning and preprocessing process are described in Section 5.3 and Section 5.4. 


\subsection{Dataset}

MovieLens is a website that gathers research data to make personalized recommendations. The MovieLens $1 \mathrm{M}$ dataset, which consists of 1 million 5-star scale ratings on 4,000 different movies provided by 6,000 different users, is used as the original dataset to carry out the comparative evaluation.

\subsection{Data Partitioning}

As discussed in the last section, given a dataset, the performance of a recommendation algorithm is affected by the rating pattern of the considered user group. The rating pattern of a user group however, could be represented by the number of rating records each user had in the dataset. Therefore, we classify all the users into different user groups according to the number of rating records they had in the MovieLens dataset. The rating records in the MovieLens dataset is then partitioned into featured data samples according to the formed user groups. Conducting comparative experiments on these featured data samples instead of the original dataset will help us better analyze how the performance of the Pawlak rough set based and the GTRS based recommendation vary on user groups with different rating patterns.

In the MovieLens dataset, about $25 \%$ of the ratings are made by users with fewer than 100 rating records, about $25 \%$ of the ratings belong to users with 100 - 200 rating records, and the other $50 \%$ are made by users with more than 200 rating records [19]. However, in the MovieLens dataset there also exist users that made an extremely large number of rating records. Therefore, although users with more than 200 rating 
Table 5.1: Five featured data samples on MovieLens

\begin{tabular}{|l|l|l|l|l|l|}
\hline Sample & Sample $_{1-50}$ & Sample $_{51-100}$ & Sample $_{101-150}$ & Sample $_{151-200}$ & Sample $_{201-250}$ \\
\hline Total ratings & 40,000 & 40,000 & 40,000 & 40,000 & 40,000 \\
\hline Total users & 1793 & 556 & 325 & 233 & 182 \\
\hline Average ratings & 22 & 71 & 123 & 172 & 220 \\
\hline
\end{tabular}

records made $50 \%$ of the ratings in the original dataset, there are only $25 \%$ of the MovieLens users in this user group. On the other hand, users with fewer than 100 rating records and users with 100-200 rating records each made $25 \%$ of the rating records, but these two user groups together consist of $75 \%$ of the MovieLens users in the original dataset.

Therefore, we further split the first and the second group into two smaller user groups. Users with fewer than 100 rating records are split into two user groups, one with a rating record range of 1-50 and the other with a rating record range of 51100. Users with 100-200 rating records are further categorized into two smaller user groups, one with a rating record range of 101-150 and the other with a rating record range of 151-200. All together the users in the MovieLens dataset are split into five featured user groups with five rating record ranges, 1-50, 51-100, 101-150, 151-200, and 201-250.

The MovieLens dataset is then partitioned into five smaller datasets according to the formed user groups. In each of these datasets, the middle 40,000 rating records are selected to form featured data samples, Sample $e_{1-50}$, Sample $e_{51-100}$, Sample S $_{101-150}$, Sample $_{151-200}$, and Sample $_{201-250}$. There are 1793, 556, 325, 233, and 182 users in these data samples, and the average number of rating records for these samples are 49, $123,153,172$, and 220. The formulation of the five featured data samples, Sample $_{1-50}$, Sample $_{51-100}$, Sample $_{101-150}$, Sample $_{151-200}$, Sample $_{201-250}$, is described in Table 5.1.

Since all the data samples are of the same size and each sample is formed with 
users with a different rating record range, the number of users in each sample are different from each other [20]. More users are involved in samples where each user makes a smaller number of rating records such as Sample $_{1-50}$, while fewer users are involved in samples where each user makes a larger number of rating records such as Sample $201-250$. The user distribution of the five featured data samples is described in Figure 5.1.

Figure 5.1: User distribution of the featured samples

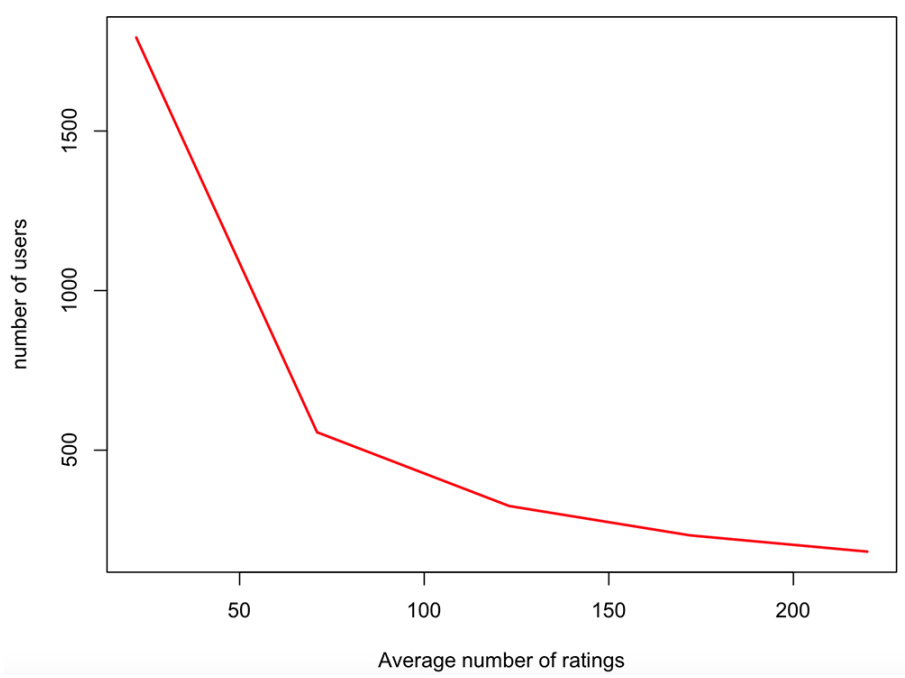

The problem of how the performance of the Pawlak rough set based and the GTRS based recommendations vary on user groups with different rating patterns could be solved by evaluating the recommendation performance of the two models on Sample $_{1-50}$ - Sample $201-250$. Moreover, we are also able to maximize the performance improvement of the GTRS model by analyzing its performance difference on the five featured data samples. 


\subsection{Data Preprocessing}

After partitioning the original dataset into featured data samples Sample ${ }_{1-50}-$ Sample $_{201-250}$, some data preprocessing are conducted on these data samples. For each data sample, the non-binary scale ratings in the original dataset are transferred into binary scale ratings. The rating records addressing ten most frequently rated movies in each data sample are selected to form equivalence classes. Besides, $80 \%$ of the rating information in each data sample are selected as training data and the remaining $20 \%$ are used as test data.

The details about the data preprocessing process is described as follows,

- Transfer into binary rating scale: We transfer the non-binary scale ratings into binary scale ratings to help the formulation of the equivalence classes. In the MovieLens dataset, a rating record of 4-5 star is considered to be a 'like' while a rating record of 1-3 star is considered to be a 'dislike'. To present the preference information in a binary scale, the rating records that are considered to be 'like' are replaced by the value ' 1 ', and the rating records that are considered to be 'dislike' are substituted by the value '-1'.

- Discover similarities among different users: For each user group, the ratings addressing ten most frequently rated movies are selected to form equivalence classes, i.e., to discover similarities among different users. We consider users with similar opinions on all considered movies to be in the same equivalence class, and each equivalence class represents a group of users with similar movie preferences.

- Divide into trainng and test data: Dividing the rating information in each of 
the featured data samples properly into training and test data is necessary in the recommendation evaluation process. In each data sample, $80 \%$ of the rating information are used as training data, and the remaining $20 \%$ are selected as test data.

After all the data partitioning and preprocessing are completed, Sample S-50 $_{1-\text { Sample }_{201-250}}$ are then used to carry out comparative experiments to examine how the performance of the Pawlak rough set based and the GTRS based recommendations vary on data samples with different rating patterns. The experimental results on the five featured data samples are further described and analyzed in the next chapter. 


\section{Chapter 6}

\section{Statistical Analysis and Comparative Evaluation}

\subsection{Accuracy and Coverage Analysis}

The training accuracy and training coverage on Sample $_{1-50}$ are described in Table 6.1 .

The Pawlak rough set model achieves an accuracy level of $100 \%$ in the prediction of each movie. In comparison, the GTRS model obtains a slightly lower accuracy level and a much higher coverage level in the prediction of each movie. For instance, the Pawlak rough set model attains an accuracy level of $100 \%$ in the prediction of Movie2, but these predictions are only available for $74.28 \%$ of the users. Under the same circumstance, the GTRS model is able to make $97.40 \%$ accurate recommendations for $90.27 \%$ of the users. In other words, the integration of the GTRS model provides us a $23.12 \%$ increment in coverage level with the cost of a slight decrement 
Table 6.1: Training results on Sample $_{1-50}$

\begin{tabular}{ccccc}
\hline Movie & GTRS accuracy & Pawlak accuracy & GTRS coverage & Pawlak coverage \\
\hline 1. & 0.9742 & 1.0000 & 0.8885 & 0.6553 \\
\hline 2. & 0.9740 & 1.0000 & 0.9027 & 0.7428 \\
\hline 3. & 0.9590 & 1.0000 & 0.9338 & 0.6805 \\
\hline 4. & 0.9817 & 1.0000 & 0.9510 & 0.8576 \\
\hline 5. & 0.9541 & 1.0000 & 0.8816 & 0.6098 \\
\hline 6. & 0.9633 & 1.0000 & 0.9107 & 0.6514 \\
\hline 7. & 0.9701 & 1.0000 & 0.8977 & 0.6609 \\
\hline 8. & 0.9548 & 1.0000 & 0.9117 & 0.6119 \\
\hline 9. & 0.9385 & 1.0000 & 0.9180 & 0.5861 \\
\hline 10. & 0.9268 & 1.0000 & 0.8268 & 0.5326 \\
\hline Avg. & 0.9597 & $\mathbf{1 . 0 0 0 0}$ & $\mathbf{0 . 9 0 2 3}$ & 0.6589 \\
\hline
\end{tabular}

in accuracy level.

In terms of the average training performance comparison between the two models, the Pawlak rough set model achieves an average accuracy level of $100 \%$ and an average coverage level of $65.89 \%$, while the GTRS model provides $95.97 \%$ accurate predictions for $90.23 \%$ of the users. Compared to the Pawlak rough set model, the GTRS model manages to make recommendations applicable for $24.34 \%$ more users with a $4.03 \%$ sacrifice in accuracy.

The test accuracy and test coverage on Sample $_{1-50}$ are described in Table 6.2.

Table 6.2: Test results on Sample $_{1-50}$

\begin{tabular}{ccccc}
\hline Movie & GTRS accuracy & Pawlak accuracy & GTRS coverage & Pawlak coverage \\
\hline 1. & 0.8228 & 0.8005 & 0.8926 & 0.6942 \\
\hline 2. & 0.8054 & 0.8195 & 0.8992 & 0.7694 \\
\hline 3. & 0.7894 & 0.7664 & 0.9107 & 0.7198 \\
\hline 4. & 0.8124 & 0.8174 & 0.9455 & 0.8868 \\
\hline 5. & 0.8324 & 0.8193 & 0.8430 & 0.6215 \\
\hline 6. & 0.7820 & 0.7386 & 0.9066 & 0.6835 \\
\hline 7. & 0.8031 & 0.7703 & 0.8777 & 0.7000 \\
\hline 8. & 0.7476 & 0.6921 & 0.8826 & 0.6388 \\
\hline 9. & 0.7677 & 0.7733 & 0.8636 & 0.6198 \\
\hline 10. & 0.6083 & 0.6375 & 0.7397 & 0.5579 \\
\hline Avg. & $\mathbf{0 . 7 7 7 0}$ & 0.7635 & $\mathbf{0 . 8 7 6 1}$ & 0.6892 \\
\hline
\end{tabular}


The test results suggest that in some cases, the GTRS model is able to make recommendations with a slightly higher accuracy level compared to the Pawlak rough set model. The GTRS model achieves a higher accuracy level the Pawlak rough set model in the predictions of Movie1, Movie3, Movie5, Movie6, Movie7, and Movie8. In other words, the GTRS model could achieve a better accuracy level than the Pawlak rough set model in 6 out of 10 cases. Furthermore, in the cases where the GTRS model outperforms the Pawlak rough set model in its accuracy level, it is still able to maintain its advantage in coverage level.

In terms of the average test performance comparison between the two models, an average accuracy level of $77.70 \%$ is obtained by the GTRS model while the Pawlak rough set model achieves an average accuracy level of $76.35 \%$. In other words, a $1.35 \%$ increment in average accuracy could be attained with the use of the GTRS model. With regard to the average coverage level, the GTRS model achieves a much higher average coverage of $87.61 \%$, while for the Pawlak rough set model, the average coverage it obtains is $68.92 \%$. The GTRS model holds a $18.69 \%$ advantage over the Pawlak rough set model in its average coverage level.

With the accuracy and coverage performance of the two models on Sample $_{1-50}$, we could conclude that on training data, the Pawlak rough set model is able to achieve a slightly higher accuracy level with a noticeable decrease in its coverage level. The GTRS model, on the other hand, manages to make recommendations for over $20 \%$ more users with a less than $4 \%$ sacrifice in its accuracy level.

More importantly, on test data, the GTRS model is not only able to make personalized recommendations applicable for more users, but also manages to predict user preferences with a slightly higher accuracy level in some of the cases. Furthermore, in the cases where the GTRS model attains a higher accuracy level than the Pawlak rough set model, it is still able to maintain its advantage in coverage level. All in 
all, compared to the Pawlak rough set model, the GTRS model is able to achieve a higher coverage level and an almost equal accuracy level on Sample $_{1-50}$.

The training and test results on Sample $_{51-100}$ - Sample $_{201-250}$ are described in Table 6.3 - Table 6.10. Similar to the training results on Sample $_{1-50}$, on all four featured data samples, the GTRS model is able to achieve a noticeable increment in its coverage level with a slight sacrifice in its accuracy level. As for test results, the GTRS model outperforms the Pawlak rough set model in its accuracy level in 6 out of 10 cases on Sample $_{51-100}$ and Sample $201-250$. On Sample Sa1-150 $_{101}$ and Sample Sa1-200, $_{151}$ it outperforms in 5 out of 10 cases. This again proves that not only could the GTRS model achieve a higher coverage level on each considered movie, but also an equal and possibly higher accuracy level on some of the considered movies.

In terms of accuracy performance variation on user groups with different rating patterns, both models achieve their highest accuracy level on Sample S1-100 $_{5}$ and their lowest accuracy level on Sample $201-250$. Both models obtain their highest cov-

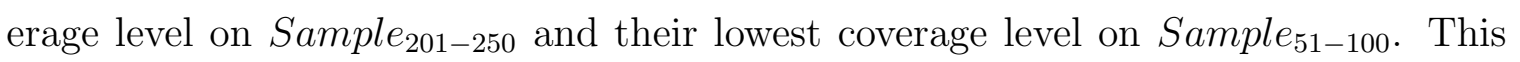
performance difference indicates that the accuracy level achieved by both models will decrease while the coverage level achieved by both models will increase on user groups with a larger number of rating records.

Overall, the GTRS model holds an advantage over the Pawlak rough set model in both average accuracy level and average coverage level. However, the level of performance improvement that the GTRS model achieves is not the same on each data sample, as the performance of the two considered models are affected by the rating pattern of the user group differently. Details about how the recommendation performance of the two models vary on the featured data samples Sample $_{1-50}$ Sample $_{201-250}$ and the reasoning behind the performance difference will be further discussed in the next section. 
Table 6.3: Training results on Sample $51-100$

\begin{tabular}{ccccc}
\hline Movie & GTRS accuracy & Pawlak accuracy & GTRS coverage & Pawlak coverage \\
\hline 1. & 0.9407 & 1.0000 & 0.9547 & 0.7756 \\
\hline 2. & 0.9743 & 1.0000 & 0.9880 & 0.8947 \\
\hline 3. & 0.9769 & 1.0000 & 0.9693 & 0.8751 \\
\hline 4. & 0.9642 & 1.0000 & 0.9809 & 0.8458 \\
\hline 5. & 0.9655 & 1.0000 & 0.9689 & 0.8613 \\
\hline 6. & 0.9735 & 1.0000 & 0.9876 & 0.8778 \\
\hline 7. & 0.9752 & 1.0000 & 0.9467 & 0.8400 \\
\hline 8. & 0.9633 & 1.0000 & 0.9462 & 0.8267 \\
\hline 9. & 0.9824 & 1.0000 & 0.9822 & 0.8973 \\
\hline 10. & 0.9800 & 1.0000 & 0.9476 & 0.8569 \\
\hline Avg. & 0.9696 & $\mathbf{1 . 0 0 0 0}$ & $\mathbf{0 . 9 6 7 2}$ & 0.8551 \\
\hline
\end{tabular}

\subsection{Accuracy and Coverage Comparison on Featured Data Samples}

Table 6.11 and Figure 6.1 describe the accuracy performance of the two models on data samples Sample $_{1-50}$ - Sample $201-250$.

The accuracy level that the Pawlak rough set model achieved ranges from $54.14 \%$ to $76.35 \%$ on the five considered user groups. For the GTRS model, the accuracy level it obtained on these data samples ranges from $54.30 \%$ to $77.70 \%$. As what we can observe from the figure, the accuracy performance of the two algorithms have a

Table 6.4: Test results on Sample S1-100 $_{10}$

\begin{tabular}{ccccc}
\hline Movie & GTRS accuracy & Pawlak accuracy & GTRS coverage & Pawlak coverage \\
\hline 1. & 0.5681 & 0.5710 & 0.9286 & 0.7946 \\
\hline 2. & 0.6920 & 0.6799 & 0.9857 & 0.9375 \\
\hline 3. & 0.7161 & 0.6977 & 0.9661 & 0.8982 \\
\hline 4. & 0.6696 & 0.6544 & 0.9857 & 0.8839 \\
\hline 5. & 0.7595 & 0.7550 & 0.9625 & 0.9000 \\
\hline 6. & 0.7256 & 0.7126 & 0.9732 & 0.8286 \\
\hline 7. & 0.6526 & 0.6624 & 0.9732 & 0.8929 \\
\hline 8. & 0.7310 & 0.7155 & 0.9268 & 0.8661 \\
\hline 9. & 0.7556 & 0.7572 & 0.9821 & 0.9464 \\
\hline 10. & 0.7094 & 0.6924 & 0.9500 & 0.8946 \\
\hline Avg. & $\mathbf{0 . 6 9 7 6}$ & 0.6898 & $\mathbf{0 . 9 6 3 4}$ & 0.8843 \\
\hline
\end{tabular}


Table 6.5: Training results on Sample S101-150 $_{10}$

\begin{tabular}{ccccc}
\hline Movie & GTRS accuracy & Pawlak accuracy & GTRS coverage & Pawlak coverage \\
\hline 1. & 0.9648 & 1.0000 & 0.9696 & 0.8631 \\
\hline 2. & 0.9636 & 1.0000 & 0.9588 & 0.8442 \\
\hline 3. & 0.9671 & 1.0000 & 0.9865 & 0.8881 \\
\hline 4. & 0.9623 & 1.0000 & 0.9919 & 0.8896 \\
\hline 5. & 0.9764 & 1.0000 & 0.9938 & 0.9288 \\
\hline 6. & 0.9769 & 1.0000 & 0.9746 & 0.9454 \\
\hline 7. & 0.9819 & 1.0000 & 0.9773 & 0.9196 \\
\hline 8. & 0.9678 & 1.0000 & 0.9819 & 0.9031 \\
\hline 9. & 0.9860 & 1.0000 & 0.9892 & 0.9369 \\
\hline 10. & 0.9398 & 1.0000 & 0.9788 & 0.8258 \\
\hline Avg. & 0.9687 & $\mathbf{1 . 0 0 0 0}$ & $\mathbf{0 . 9 8 0 2}$ & 0.8947 \\
\hline
\end{tabular}

tight competition with each other on all five data samples. Since the performance difference between the two modes ranges from $0.16 \%$ to $1.35 \%$, it is fair to conclude that both models achieve an almost equal accuracy level on each of the considered data samples.

In terms of accuracy performance variation on user groups with different rating patterns, the accuracy level of the two considered models both decreases when making recommendations for user groups with a larger number of rating records. For instance, the Pawlak rough set model achieves an accuracy level of $76.35 \%$ on Sample $_{1-50}$, while on Sample $201-250$ it achieves an accuracy level of $54.14 \%$. For the GTRS model, the accuracy level it obtains on Sample $_{1-50}$ is $77.70 \%$, while on Sample $_{201-250}$ it obtains

Table 6.6: Test results on Sample S $_{101-150}$

\begin{tabular}{ccccc}
\hline Movie & GTRS accuracy & Pawlak accuracy & GTRS coverage & Pawlak coverage \\
\hline 1. & 0.6457 & 0.6559 & 0.9769 & 0.9154 \\
\hline 2. & 0.6801 & 0.6579 & 0.9615 & 0.9000 \\
\hline 3. & 0.5979 & 0.5984 & 0.9231 & 0.8846 \\
\hline 4. & 0.6131 & 0.5925 & 0.9538 & 0.9077 \\
\hline 5. & 0.6562 & 0.6423 & 0.9846 & 0.9462 \\
\hline 6. & 0.6228 & 0.6173 & 0.9769 & 0.9385 \\
\hline 7. & 0.6761 & 0.6525 & 0.9764 & 0.9077 \\
\hline 8. & 0.5885 & 0.5918 & 0.9600 & 0.9308 \\
\hline 9. & 0.5920 & 0.5953 & 0.9631 & 0.9462 \\
\hline 10. & 0.4879 & 0.4932 & 0.9508 & 0.8769 \\
\hline Avg. & $\mathbf{0 . 6 1 6 0}$ & 0.6097 & $\mathbf{0 . 9 6 2 7}$ & 0.9154 \\
\hline
\end{tabular}


Table 6.7: Training results on Sample S151-200 $_{10}$

\begin{tabular}{ccccc}
\hline Movie & GTRS accuracy & Pawlak accuracy & GTRS coverage & Pawlak coverage \\
\hline 1. & 0.9748 & 1.0000 & 0.9968 & 0.9385 \\
\hline 2. & 0.9716 & 1.0000 & 0.9984 & 0.9321 \\
\hline 3. & 0.9856 & 1.0000 & 0.9923 & 0.9538 \\
\hline 4. & 0.9743 & 1.0000 & 0.9891 & 0.9412 \\
\hline 5. & 0.9764 & 1.0000 & 0.9938 & 0.9288 \\
\hline 6. & 0.9748 & 1.0000 & 0.9984 & 0.9390 \\
\hline 7. & 0.9754 & 1.0000 & 0.9896 & 0.9449 \\
\hline 8. & 0.9732 & 1.0000 & 0.9979 & 0.9348 \\
\hline 9. & 0.9860 & 1.0000 & 0.9892 & 0.9369 \\
\hline 10. & 0.9757 & 1.0000 & 0.9904 & 0.9273 \\
\hline Avg. & 0.9780 & $\mathbf{1 . 0 0 0 0}$ & $\mathbf{0 . 9 7 8 0}$ & 0.9377 \\
\hline
\end{tabular}

an accuracy level of $54.30 \%$.

One reason accounting for this performance difference is that given a dataset, there are always more users in user groups with a smaller number of rating records than in user groups with a larger number of rating records. The equivalence classes formed based on data samples like Sample $_{201-250}$ are generally smaller than the ones formed on data samples like Sample $_{1-50}$. With fewer similiar users to learn rating patterns from, the predictions of user preferences become less accurate. The other reason accounting for this performance decrease is that the coverage levels on user groups with a larger number of rating records are normally higher than user groups with a smaller number of rating records. This means on user groups with a larger

Table 6.8: Test results on Sample S $_{151-200}$

\begin{tabular}{ccccc}
\hline Movie & GTRS accuracy & Pawlak accuracy & GTRS coverage & Pawlak coverage \\
\hline 1. & 0.5482 & 0.5374 & 0.9348 & 0.9130 \\
\hline 2. & 0.4973 & 0.5041 & 0.9891 & 0.9674 \\
\hline 3. & 0.5439 & 0.5432 & 0.9761 & 0.9739 \\
\hline 4. & 0.5684 & 0.5695 & 0.9804 & 0.9077 \\
\hline 5. & 0.6562 & 0.6423 & 0.9846 & 0.9462 \\
\hline 6. & 0.6166 & 0.6170 & 0.9717 & 0.9500 \\
\hline 7. & 0.5091 & 0.5085 & 0.9739 & 0.9374 \\
\hline 8. & 0.6202 & 0.6213 & 0.9783 & 0.9308 \\
\hline 9. & 0.5805 & 0.5725 & 0.9130 & 0.8804 \\
\hline 10. & 0.5333 & 0.5345 & 0.9630 & 0.9413 \\
\hline Avg. & $\mathbf{0 . 5 6 7 4}$ & 0.5651 & $\mathbf{0 . 9 6 6 5}$ & 0.9348 \\
\hline
\end{tabular}


Table 6.9: Training results on Sample $201-250$

\begin{tabular}{ccccc}
\hline Movie & GTRS accuracy & Pawlak accuracy & GTRS coverage & Pawlak coverage \\
\hline 1. & 0.9648 & 1.0000 & 0.9696 & 0.8631 \\
\hline 2. & 0.9773 & 1.0000 & 0.9979 & 0.9418 \\
\hline 3. & 0.9678 & 1.0000 & 0.9819 & 0.9031 \\
\hline 4. & 0.9493 & 1.0000 & 0.9861 & 0.8836 \\
\hline 5. & 0.9794 & 1.0000 & 0.9979 & 0.9397 \\
\hline 6. & 0.9710 & 1.0000 & 0.9938 & 0.9178 \\
\hline 7. & 0.9720 & 1.0000 & 0.9760 & 0.8945 \\
\hline 8. & 0.9681 & 1.0000 & 0.9836 & 0.8734 \\
\hline 9. & 0.9398 & 1.0000 & 0.9788 & 0.8258 \\
\hline 10. & 0.9738 & 1.0000 & 0.9904 & 0.9205 \\
\hline Avg. & 0.9663 & $\mathbf{1 . 0 0 0 0}$ & $\mathbf{0 . 9 8 5 6}$ & 0.8963 \\
\hline
\end{tabular}

number of rating records, preference predictions are made available for more users. However, both accuracy and coverage have to be considered in combination with each other, as an increase in one attribute will result in a decrease in the other. Therefore, as recommendations could be made for more users on user groups with a larger number of rating records, these recommendations are not as accurate as the ones on user groups with a smaller number of rating records.

The coverage performance of the two models on Sample $_{1-50}-$ Sample $_{201-250}$ are described in Table 6.12 and Figure 6.2.

Different from what we have discussed in the case of accuracy, the GTRS model achieves a noticeable improvement in coverage level over the Pawlak rough set model

Table 6.10: Test results on Sample $201-250$

\begin{tabular}{ccccc}
\hline Movie & GTRS accuracy & Pawlak accuracy & GTRS coverage & Pawlak coverage \\
\hline 1. & 0.4412 & 0.4246 & 0.9444 & 0.9167 \\
\hline 2. & 0.4586 & 0.4512 & 0.9722 & 0.9583 \\
\hline 3. & 0.5206 & 0.5197 & 0.9861 & 0.9306 \\
\hline 4. & 0.4976 & 0.4953 & 0.9833 & 0.9556 \\
\hline 5. & 0.6639 & 0.6734 & 0.9842 & 0.9296 \\
\hline 6. & 0.5755 & 0.5691 & 0.9806 & 0.9583 \\
\hline 7. & 0.5921 & 0.5989 & 0.9722 & 0.9028 \\
\hline 8. & 0.5727 & 0.5730 & 0.9528 & 0.9167 \\
\hline 9. & 0.5920 & 0.5953 & 0.9631 & 0.9462 \\
\hline 10. & 0.5160 & 0.5135 & 0.9528 & 0.9064 \\
\hline Avg. & $\mathbf{0 . 5 4 3 0}$ & 0.5414 & $\mathbf{0 . 9 6 9 2}$ & 0.9321 \\
\hline
\end{tabular}


Table 6.11: Accuracy of the two prediction algorithms on the featured data samples

\begin{tabular}{cccccc}
\hline Model & Sample $_{1-50}$ & Sample $_{51-100}$ & Sample $_{101-150}$ & Sample $_{151-200}$ & Sample $_{201-250}$ \\
\hline GTRS & 0.7770 & 0.6976 & 0.6160 & 0.5674 & 0.5430 \\
\hline Pawlak & 0.7635 & 0.6898 & 0.6097 & 0.5651 & 0.5414 \\
\hline
\end{tabular}

Figure 6.1: Accuracy of the two prediction algorithms on the featured data samples

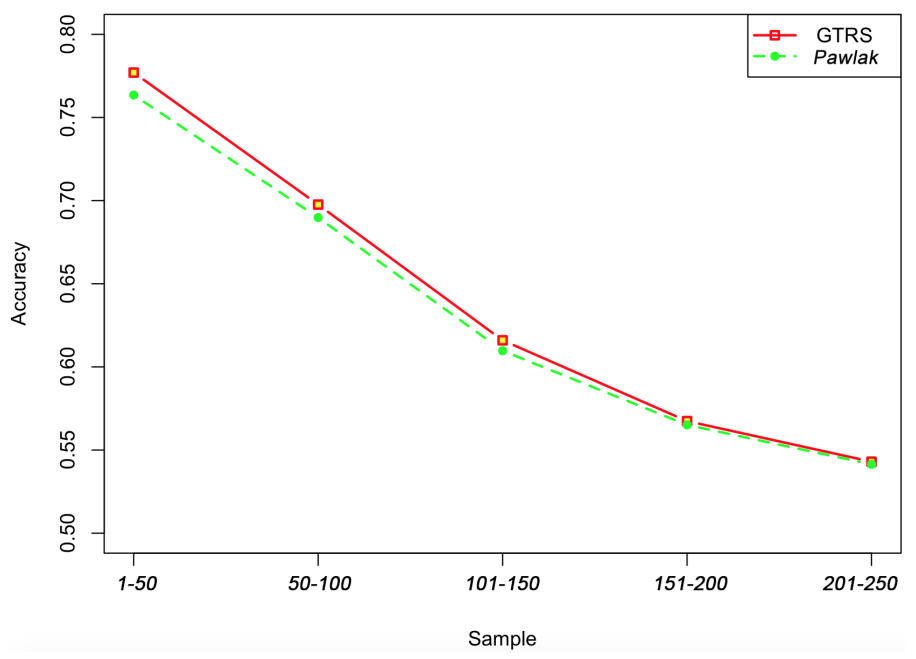

Table 6.12: Coverage of the two prediction algorithms on the featured data samples

\begin{tabular}{cccccc}
\hline Model & Sample $_{1-50}$ & Sample $_{51-100}$ & Sample $_{101-150}$ & Sample $_{151-200}$ & Sample $_{201-250}$ \\
\hline GTRS & 0.8761 & 0.9634 & 0.9627 & 0.9665 & 0.9692 \\
\hline Pawlak & 0.6892 & 0.8843 & 0.9154 & 0.9348 & 0.9321 \\
\hline
\end{tabular}

Figure 6.2: Coverage of the two prediction algorithms on the featured data samples

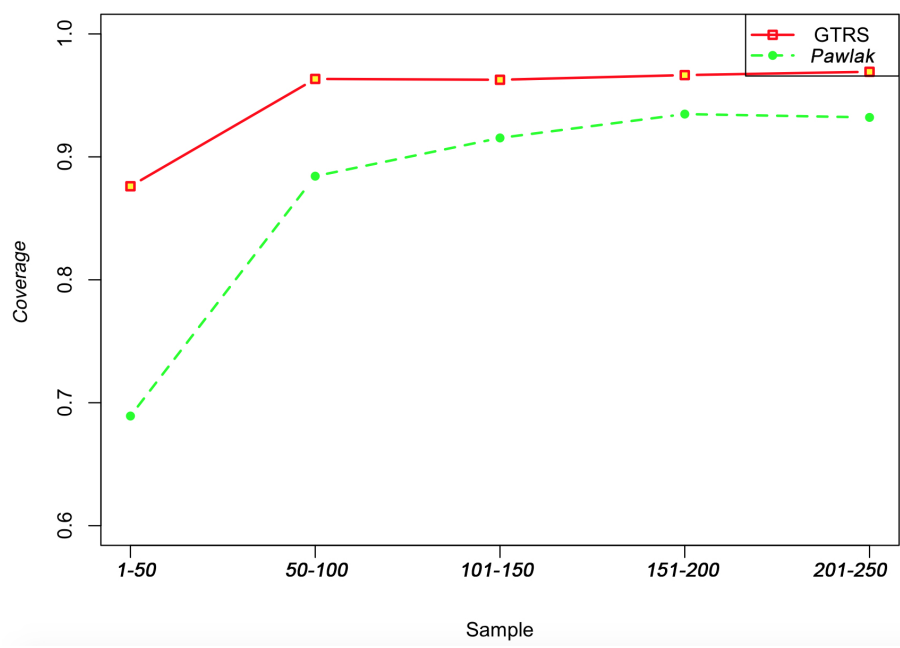


on all five considered user groups Sample $_{1-50}-$ Sample $_{201-250}$. This means the GTRS model is able to make preference predictions available for more users no matter how many rating records each user had in the original database.

In terms of coverage performance variation on user groups with different rating patterns, the coverage level of both models will increase when making predictions for user groups with more rating records. As the Pawlak rough set model and the GTRS model achieve their lowest coverage level at $87.61 \%$ and $68.92 \%$ respectively on Sample $_{1-50}$, and obtain their highest coverage level at $96.92 \%$ and $93.21 \%$ respectively on Sample $201-250$. In other words, both models are able to adjust and make recommendations applicable for more users on user groups with a larger number of rating records.

The GTRS model holds a $18.89 \%$ advantage in coverage level over the Pawlak rough set model on Sample $_{1-50}$, however, this advantage drops to $3.71 \%$ on Sample $_{201-250}$. One reason accounting for this performance difference is also that there are a lot more users in user groups with a smaller number of rating records like Sample $_{1-50}$, and a lot fewer users in user groups with a larger number of rating records like Sample $201-250$. As user groups with smaller number of rating records normally consist of more users, the equivalence classes formed on these user groups are generally larger. The adjustments in the threshold pair values will therefore have a bigger impact on data samples with larger equivalence classes compared to the ones with smaller equivalence classes. The GTRS model manipulates the coverage level by adjusting the threshold pair values. Therefore, the increment it brought in coverage level is bigger on user groups with more users like $S_{a m p l e_{1-50}}$, and smaller on user groups with fewer users like Sample $201-250$. Moreover, although its advantage in coverage level is not as obvious

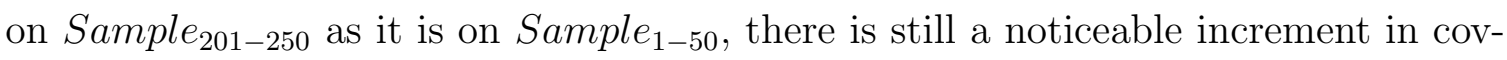
erage level on each of the considered data samples. 
With the accuracy and coverage analysis on the considered data samples, we could summarize that the GTRS model is able to improve the overall quality of the Pawlak rough set based recommendations. Since through the incorporation of the GTRS model, not only could a recommender system make personalized predictions applicable for more users, but also recommend users' preferences with an equal level of accuracy.

With regard to the performance variation on user groups with different rating patterns, we could conclude that the coverage level of both models will increase while the accuracy level will decrease on user groups with a larger number of rating records. Moreover, although the GTRS model achieves an almost equal accuracy level with the Pawlak rough set model on all the considered data samples, the advantage it holds in coverage level is bigger on user groups with a smaller number of rating records. There-

fore, the overall performance improvement brought by the GTRS model is the bigger on user groups with a smaller number of rating records. This advantage is not as obvious on user groups with a larger number of rating records, as the adjustments in threshold levels have a bigger impact on data samples with larger equivalence classes. This performance difference further indicates that compared to the Pawlak rough set model, the GTRS model might be a better solution when preference predictions need to be made for users that make a smaller number of rating records.

\subsection{Decision Support Metric Analysis}

In many cases, a system designer chooses the appropriate recommendation algorithm from a set of candidate algorithms by evaluating each approach with the chosen 
metrics that are suitable for the overall purpose of the system [20]. Different evaluation metrics have been proposed to help a system designer in the process of choosing the appropriate algorithm, and each of these evaluation metrics provide us a different aspect in the performance evalulation of a recommendation algorithm [18].

As discussed in the last section, when the performance of different recommendation algorithms are evaluated and compared with each other, the results provided by different evaluation metrics might be different. Each evaluation metric reflects a certain performance aspect of the considered recommendation algorithm, therefore, it is necessary to incorporate multiple evaluation metrics in the comparison between the Pawlak rough set based and the GTRS based recommendations.

Besides the accurcy and the coverage level of its recommendations, the manner of how these recommendations are delivered to the users is also an important performance aspect in the evaluation of a recommendation algorithm [12]. The ratio of correctly and incorrectly recommended items provide a further insight into the recommendation delivery manner of a recommendation algorithm, and could be measured through the use of decision-support metrics. Decision-support metrics evaluate the classification ability of a recommendation algorithm by calculating the frequency where correct and incorrect decisions on a considered item are made [23]. The decision on a considered item is correct if it is recommended and preferred by the considered user, or if it is not recommended and not preferred by the considered user. Otherwise, the decision on this item is considered incorrect.

Two different decision support metrics, precision-recall, and ROC curve-based metrics are employed to carry out the performance evaluation of the two models [11]. Precision measures the probability that a recommended item is preferred, and recall defines the ratio of prefered and recommended item to all the items in the preferred item set. The ROC curve-based metrics compute the ratio of preferred and not 
Table 6.13: Categorization of recommendation decisions

\begin{tabular}{ccc}
\hline & Recommended & Not recommended \\
\hline Preferred & True positive (TP) & False negative (FN) \\
\hline Not preferred & False positive (FP) & True negative (TN) \\
\hline
\end{tabular}

preferred recommended items to all the items in the preferred item set. These two metrics provide us different yet complementary aspects of the classification ability of a recommendation algorithm. Precision-recall emphasizes the ratio of recommended items that are are preferred by the considered users, while ROC curve-based metrics focus more on the proportion of recommended items that are not preferred by the considered users [19].

\subsubsection{Precision-recall Analysis}

Precision-recall is one of the most widely used decision support metrics in recommendation performance evaluation $[30,35,8,39]$. To carry out precision-recall analysis, the two criteria, precision and recall need to be addressed.

A confusion matrix is needed to compute these two attributes. Table 6.13 is an example of a confusion matrix, and the four cells represents four different categories of the possibile results of any recommendation decision [17]. The item set that each user rated for is classified into two categories, preferred and not preferred, where for each user in the dataset, preferred represents the set of items he or she rates positively, and not preferred represents the set of items he or she rates negatively. Likewise, for each user, the item set is also classified into the categories of recommended and not recommended, based on whether or not an item is recommended to him or her. 
With the above classification matrix, all recommendation decisions are classified into the following four categories: true positive (TP), true negative (TN), false positive (FP), and false negative (FN). True positive (TP) represents a set of decisions where a recommended item is preferred by the considered user, while true negative (TN) describes a set of decisions where a not preferred item is not recommended to the considered user. False negative (FN) illustrates a set of decisions where a preferred item is not recommended to the considered user, while false positive (FP) represents a set of decisions where a recommended item is not preferred by the considered user.

Precision is defined as the ratio of recommended and preferred items to all the items in the recommended item set. In other words, it represents the probability that a recommended item is preferred by the considered user. Recall is defined as the ratio of recommended and preferred items to all the items in the preferred item set [19]. In other words, it describes the probability where a preferred item is indeed recommended to the considered user.

By going through the confusion matrix and classifying all the recommendation decisions into the appropriate category, the metric of precision and the metric of recall are calculated using the following equations [19],

$$
\begin{gathered}
\text { precision }=\frac{T P}{T P+F P} \\
\text { recall }=\frac{T P}{T P+F N}
\end{gathered}
$$

In the rough set model, with the determination of the three rough set regions, the metric of precision and the metric of recall are defined using the following equations,

$$
\text { precision }=\frac{\left|\left(P O S_{\alpha, \beta}(C) \cap C\right)\right|}{\left|\left(P O S_{\alpha, \beta}(C) \cap C\right) \cup\left(P O S_{\alpha, \beta}(C) \cap C^{c}\right)\right|}
$$




$$
\text { recall }=\frac{\left|P O S_{\alpha, \beta}(C) \cap C\right|}{\left|\left(P O S_{\alpha, \beta}(C) \cap C\right) \cup\left(N E G_{\alpha, \beta}(C) \cap C\right)\right|}
$$

To carry out precision-recall analysis, the precision and recall level achieved by the two considered models are calculated on the five featured data samples. Each pair of the calculated values of (precision, recall) represents the algorithm's classification ability on a user group with a certain rating pattern. Similar to accuracy and coverage, the metric of precision and the metric of recall are always evaluated in combination with each other. The goal of the precision-recall analysis is to achieve a higher precision level without sacrificing the recall level, in other words, to optimize the two attributes at the same time [17].

The (precision, recall) pairs that the two considered models achieve on the five featured data samples are calculated using Eq 6.34 and Eq 6.35 [19]. With the calculated (precision, recall) pairs, we are able to analyze and compare the classification ability of the two considered models on data samples with different rating patterns. The decision on which algorithm is better will be easy if one algorithm outperforms the other on both of the attributes. However, when an algorithm is winning in one attribute and losing in the other, the decision on the winning algorithm depends on the overall purpose of the system. For instance, an algorithm with a better overall recall performance is preferred if the goal of the system is to "recommend some good items". Likewise, an algorithm with a better overall precision performance is preferred when the goal of the system is to "recommend all good items" [19]. 


\subsubsection{Precision-recall Comparison on Featured Data Samples}

The (precision, recall) pairs achieved by the two models on the featured data samples Sample $_{1-50}$ - Sample $_{201-250}$ are described in Table 6.14.

The recall level achieved by the Pawlak rough set model ranges from $14.45 \%$ to $32.92 \%$, while the precision level ranges from $29.04 \%$ to $52.07 \%$ on the five featured data samples. On the other hand, the recall level achieved by the GTRS model ranges from $13.21 \%$ to $33.31 \%$, while the precision level ranges from $30.55 \%$ to $52.65 \%$. The GTRS model achieves a higher precision level on all five data samples and a higher recall level on 3 out of 5 data samples, Sample ${ }_{51-100}$, Sample ${ }_{101-150}$, Sample Sa1-250. $_{2}$. In other words, the GTRS model has a better classification ability compared to the Pawlak rough set model on these data samples. As it is able to achieve a higher precision level in all cases, and a higher recall level in some of the cases.

In terms of the performance variations on user groups with different rating patterns, for both models, the precision level decreases while the recall level increases on user groups with a larger number of rating records. Therefore, both models are able to cover more preferred movies in their recommendations when more rating records are made by users in the considered user group. However, as more recommendations are made and more preferred movies are covered on user groups with more rating records, the probability of a recommended movie is preferred by the considered user becomes lower.

Table 6.14: Precision and recall of the two prediction algorithms on the featured data samples

\begin{tabular}{ccccc}
\hline Model & GTRS precision & Pawlak precision & GTRS recall & Pawlak recall \\
\hline Sample $_{1-50}$ & 0.5265 & 0.5207 & 0.1321 & 0.1445 \\
\hline Sample $_{51-100}$ & 0.5073 & 0.4877 & 0.2625 & 0.2563 \\
\hline Sample $_{101-150}$ & 0.4064 & 0.4048 & 0.3156 & 0.2957 \\
\hline Sample $_{151-200}$ & 0.3889 & 0.3806 & 0.3321 & 0.3389 \\
\hline Sample $_{201-250}$ & 0.3055 & 0.2904 & 0.3331 & 0.3292 \\
\hline
\end{tabular}


The precision-recall analysis suggest that compared to the Pawlak rough set model, the GTRS model has a better classification aility compared to the Pawlak rough set model in the majority of cases. Since the GTRS model is able to obtain a higher precision level on each of the featured data samples, as well as a higher recall level on 3 out of 5 featured data samples. Therefore, given the condition that the same number of recommendations are generated, the recommendations made by the GTRS model are more likely to be in the preferred movie set on all the considered user groups. Moreover, the GTRS model based recommendations are also able to cover more preferred movies in the preferred movie set on 3 out of 5 user groups.

\subsubsection{Receiver Operating Curve Based Metrics Analysis}

ROC curve-based metrics computes the ratio of correct and incorrect recommendation decisions by calculating the metrics of true positive rate (TPR) and false positve rate $(F P R)[45,21]$. The two metrics are both calculated using the same confusion matrix in Table 6.13. TPR, which is indicated in Eq 6.34, calculates the ratio of recommended and preferred items to all the items in the preferred item set. FPR, which is described in Eq 6.35, measures the ratio of recommended and not preferred items to all the items in the not preferred item set.

$$
\begin{aligned}
& T P R=\frac{T P}{T P+F N} \\
& F P R=\frac{F P}{F P+T N}
\end{aligned}
$$

In the rough set model, once the three rough set regions are determined, the metric of $T P R$ and the metric of $F P R$ are defined using the following equations, 


$$
\begin{gathered}
T P R=\frac{\left|P O S_{\alpha, \beta}(C) \cap C\right|}{\mid\left(P O S_{\alpha, \beta}(C) \cap C \cup\left(N E G_{\alpha, \beta}(C) \cap C\right) \mid\right.} \\
F P R=\frac{\left|\left(P O S_{\alpha, \beta}(C) \cap C^{c}\right)\right|}{\left|\left(P O S_{\alpha, \beta}(C) \cap C^{c}\right) \cup\left(N E G_{\alpha, \beta}(C) \cap C^{c}\right)\right|}
\end{gathered}
$$

The overall goal of ROC analysis is to recommend preferred items, as well as to avoid returning not preferred items. Therefore, different from precision-recall, the optimization of the ROC curve-based metrics could be achieved by maximizing TPR and minimizing FPR [41].

Although both metrics measure the ratio of preferred and recommended items in the preferred item set, precision-recall focuses on the proportion of recommended items that are preferred by the considered user group. The ROC curve-based metrics on the other hand, emphasize the proportion of not preferred items that end up being recommended to the considered user group [20].

\subsubsection{ROC Curve-based Metrics Comparison on Featured Data Samples}

The (TPR, FPR) pairs achieved by the two models on the featured data samples Sample $_{1-50}$ - Sample $201-250$ are described in Table 6.15.

Table 6.15: TPR and FPR of the two prediction algorithms on the featured data samples

\begin{tabular}{ccccc}
\hline Model & GTRS TPR & Pawlak TPR & GTRS FPR & Pawlak FPR \\
\hline Sample $_{1-50}$ & 0.1321 & 0.1445 & 0.0895 & 0.1328 \\
\hline Sample $_{51-100}$ & 0.2625 & 0.2563 & 0.1340 & 0.1454 \\
\hline Sample $_{101-150}$ & 0.3156 & 0.2957 & 0.2078 & 0.2110 \\
\hline Sample $_{151-200}$ & 0.3321 & 0.3389 & 0.2711 & 0.2689 \\
\hline Sample $_{201-250}$ & 0.3331 & 0.3292 & 0.3095 & 0.3145 \\
\hline
\end{tabular}


The TPR achieved by the Pawlak rough set model ranges from $14.45 \%$ to $32.92 \%$, while the FPR ranges from $13.28 \%$ to $31.45 \%$ on the five featured data samples. On the other hand, the TPR achieved by the GTRS model ranges from $13.21 \%$ to $33.31 \%$ while the FPR ranges from $8.95 \%$ to $30.95 \%$ on the five featured data samples. The GTRS model achieves a higher TPR level on 3 out of 5 data samples, Sample $_{51-100}$, Sample $_{101-150}$, Sample $_{201-250}$, and a lower FPR level on 4 out of 5 data samples, Sample $1_{1-50}$, Sample $_{51-100}$, Sample $_{101-150}$, Sample $_{201-250}$.

In terms of performance variations on user groups with different rating patterns, the FPR and TPR levels of the two models both increase on user groups with a larger number of the rating records. For a given user group, as more rating records are made by each user, both models are able to generate more movie recommendations. As a result, these recommendations include not only more preferred movies in the preferred movie set, but also more not preferred movies in the not preferred movie set.

The ROC curve-based metrics suggest that compared to the Pawlak rough set model, the GTRS model achieves a lower FPR on 4 out of 5 data samples, and a higher TPR on 3 out of 5 data samples. This again proves that the GTRS model has a better classification aility compared to the Pawlak rough set model in the majority of cases. Therefore, given the condition that the same number of recommendations are made, the GTRS model could not only include fewer not preferred movies in its recommendations on 4 out of 5 user groups, but also cover more preferred movies on 3 out of 5 user groups. 


\subsection{Overall Performance Analysis and Comparison}

The experimental results above describe the recommendation performance of the Pawlak rough set model and the GTRS model on the five featured data samples Sample $_{1-50}$ - Sample $201-250$. With the comparative evaluations using the three chosen evaluation metrics, accuracy and coverage, precision-recall, and ROC curvebased metrics, we could conclude that,

- According to the accuracy and coverage metrics, the GTRS model is able to make the Pawlak rough set based recommendations applicable for more users while maintaining an almost equal accuracy level on the five featured data samples Sample $_{1-50}$ - Sample $201-250 ;$

- According to the precision-recall metric and ROC curve-based metrics, the GTRS model has a better classification ability compared to the Pawlak rough set model in the majority of cases;

- According to the precision-recall metric, the recommendations made by the GTRS model are more likely to be in the preferred movie set on all the considered user groups. Moreover, it is also able to cover more preferred movies in the preferred movie set on 3 out of 5 user groups;

- According to the ROC curve-based metrics, the GTRS model could not only include fewer not preferred movies in its recommendations on 4 out of 5 user groups, but also cover more preferred movies on 3 out of 5 user groups;

- In terms of performance variations on user groups with different rating patterns, the accuracy level and the precision level of the two models will decrease while 
the coverage, recall, $T P R$ and $F P R$ levels will increase on user groups with a larger number of rating records;

- The GTRS model holds a bigger advantage over the Pawlak rough set model especially on user groups with a smaller number of rating records such as Sample $_{1-50}$ and Sample $_{51-100}$. This advantage becomes less obvious on user groups with a larger number of rating records as the Pawlak rough set model is able to improve its performance as more rating records are added to the database. 


\section{Chapter 7}

\section{Conclusions and Discussion}

The GTRS model improves on the quality of the Pawlak rough set based recommendations by formulating a competitive game between two of the prominent recommendation evaluation metrics, accuracy and coverage. With the GTRS model, a threshold pair $(\alpha, \beta)$ will be attained once a tradeoff is achieved between the two considered evaluation metrics. Approximating user preferences with the calculated GTRS threshold pair makes the Pawlak rough set based recommendations applicable for more users, which helps with its practical application.

As the performance of a recommendation algorithm may be affected by the rating patterns of the users in the considered dataset, comparative experiments are carried out on five different data samples to evaluate how the quality of the Pawlak rough set based and the GTRS based recommendations vary on user groups with different rating patterns. The two models are used to predict user preferences on five featured data samples respectively, and their recommendation quality on each sample are evaluated and compared with each other. To make sure a thorough comparison 
is achieved, beside accuracy and coverage, two decision support metrics, precisionrecall, and ROC curve-based metrics are used to examine the classification ability of the two models.

The experimental results suggest that the GTRS model holds an advantage over the Pawlak rough set model in coverage level and achieves an almost equal performance in accuracy level on each of the considered data sample. Moreover, the GTRS model has an overall better permance in its precision-recall and ROC curve-based metrics levels on 3 out of 5 considered user groups, which means it has a better classification ability compared to the Pawlak rough set model in the majority of cases.

Although the GTRS model achieves an overall better performance compared to the Pawlak rough set model on every considered data sample, the performance improvement on each sample is not the same as the performance of the two models are affected by the rating pattern of the user group differently. The advantage that the GTRS model holds over the Pawlak rough set model is bigger on user groups with a smaller number of rating records, and is not as obvious on user groups with a larger number of rating records. One reason accounting for this performance difference is that the equivalence classes formed on user groups with a smaller number of rating records are generally larger than those on user groups with a larger number of rating records. The GTRS model manipulates the accuracy and coverage level by adjusting the thresholds values, and these adjustments have a bigger impact on data samples with larger equivalence classes. More reasonings behind the performance difference could be addressed by using the GTRS threshold pair attained on one user group to predict user preferences on another user group. Conducting these cross recommendations among different data samples in future research will provide us a futher insight into the relationship between the rating pattern of the user group and the performance of the recommendation algorithm. 
Not being able to significantly increase its performance as more rating records are added to the database might be a limitation of the GTRS based recommendations. Although it still achieves an overall better performance compared to the Pawlak rough set model, the overall performance of the GTRS model is not as competitive as some other data mining models on user groups with a larger number of rating records. Therefore, it is not the best algorithm to predict user preferences on datasets where users made a larger number of rating records compared to some other model-based techniques such as the latent semantic model. However, some of these techniques require a large number of user rating records in the model building process, and are not able to perform if the provided user rating records are not enough. The GTRS model on the other hand, is able to make moderately accurate recommendations even on data samples with fewer rating records stored in the database.

The ultimate success of any recommender system is having the ability to make highly accurate recommendations for almost every user in the system, however, this may not always be possible in real world situations. Especially, it could be hard for a recommendation algorithm to perform well when fewer rating records are stored in the database. Therefore, incorporating the GTRS model is an effective solution to make personalized recommendation on datasets formed by users that make a smaller number of rating records. 


\section{References}

[1] Gediminas Adomavicius and YoungOk Kwon. New recommendation techniques for multicriteria rating systems. IEEE Intelligent Systems, 22(3):48-55, 2007.

[2] Gediminas Adomavicius, Ramesh Sankaranarayanan, Shahana Sen, and Alexander Tuzhilin. Incorporating contextual information in recommender systems using a multidimensional approach. ACM Transactions on Information Systems, 23(1):103-145, 2005.

[3] Gediminas Adomavicius and Alexander Tuzhilin. Toward the next generation of recommender systems: A survey of the state-of-the-art and possible extensions. IEEE Transactions on Knowledge and Data Engineering, 17(6):734-749, 2005.

[4] Asim Ansari, Skander Essegaier, and Rajeev Kohli. Internet recommendation systems. Journal of Marketing Research, 37(3):363-375, 2000.

[5] Nouman Azam and JingTao Yao. Analyzing uncertainties of probabilistic rough set regions with game-theoretic rough sets. International Journal of Approximate Reasoning, 55(1):142-155, 2014.

[6] Nouman Azam and JingTao Yao. Game-theoretic rough sets for recommender systems. Knowledge-Based Systems, 72:96-107, 2014. 
[7] Nouman Azam and JingTao Yao. Multiple criteria decision analysis with gametheoretic rough sets. In Proceedings of the International Conference on Rough Sets and Knowledge Technology. 2012, pp.187-192.

[8] Chumki Basu, Haym Hirsh, and William Cohen. Recommendation as classification: Using social and content-based information in recommendation. In Proceedings of the Fifteenth National Conference on Artificial Intelligence. 1998, pp.714-720.

[9] Punam Bedi and Suruchi Chawla. Use of fuzzy rough set attribute reduction in high scent web page recommendations. In Proceedings of the International Workshop on Rough Sets, Fuzzy Sets, Data Mining, and Granular-Soft Computing. 2009, pp.192-200.

[10] Jesús Bobadilla, Fernando Ortega, Antonio Hernando, and Abraham Gutiérrez. Recommender systems survey. Knowledge-Based Systems, 46:109-132, 2013.

[11] Andrew P. Bradley. The use of the area under the roc curve in the evaluation of machine learning algorithms. Pattern Recognition, 30(7):1145-1159, 1997.

[12] John S. Breese, David Heckerman, and Carl Kadie. Empirical analysis of predictive algorithms for collaborative filtering. In Proceedings of the Fourteenth Conference on Uncertainty in Artificial Intelligence. 1998, pp.43-52.

[13] Robin Burke. Hybrid recommender systems: Survey and experiments. User Modeling and User-adapted Interaction, 12(4):331-370, 2002.

[14] Sonny Han Seng Chee, Jiawei Han, and Ke Wang. Rectree: An efficient collaborative filtering method. In Proceedings of the Third International Conference on Big Data Analytics and Knowledge Discovery. 2001, pp.141-151. 
[15] Ricardo Colomo-Palacios, Israel González-Carrasco, José Luis López-Cuadrado, and Ángel García-Crespo. Resyster: A hybrid recommender system for scrum team roles based on fuzzy and rough sets. International Journal of Applied Mathematics and Computer Science, 22(4):801-816, 2012.

[16] Paolo Cremonesi, Roberto Turrin, Eugenio Lentini, and Matteo Matteucci. An evaluation methodology for collaborative recommender systems. In Proceedings of the International Conference on Automated solutions for Cross Media Content and Multi-channel Distribution. 2008, pp.224-231.

[17] Félix Hernández del Olmo and Elena Gaudioso. Evaluation of recommender systems: A new approach. Expert Systems with Applications, 35(3):790-804, 2008.

[18] Nathaniel Good, J. Ben Schafer, Joseph A. Konstan, Al Borchers, Badrul Sarwar, Jon Herlocker, and John Riedl. Combining collaborative filtering with personal agents for better recommendations. In Proceedings of the Sixteenth National Conference on Artificial Intelligence. 1999, pp.439-446.

[19] Asela Gunawardana and Guy Shani. A survey of accuracy evaluation metrics of recommendation tasks. Journal of Machine Learning Research, 10:2935-2962, 2009.

[20] Asela Gunawardana and Guy Shani. Evaluating recommender systems. In Recommender Systems Handbook. 2015, pp.265-308.

[21] James A. Hanley and Barbara J. McNeil. The meaning and use of the area under a receiver operating characteristic (roc) curve. Radiology, 143(1):29-36, 1982.

[22] Joseph P. Herbert and JingTao Yao. Game-theoretic rough sets. Fundamenta Informaticae, 108(3-4):267-286, 2011. 
[23] Jonathan L. Herlocker, Joseph A. Konstan, Loren G. Terveen, and John T. Riedl. Evaluating collaborative filtering recommender systems. ACM Transactions on Information Systems, 22(1):5-53, 2004.

[24] Chongben Huang and Songjie Gong. Employing rough set theory to alleviate the sparsity issue in recommender system. In Proceedings of the 2008 International Conference on Machine Learning and Cybernetics. 2008, pp.1610-1614.

[25] Zan Huang, Daniel Zeng, and Hsinchun Chen. A comparison of collaborativefiltering recommendation algorithms for e-commerce. IEEE Intelligent Systems, 22(5):68-78, 2007.

[26] Tomoko Kashima, Shimpei Matsumoto, and Hiroaki Ishii. Decision support system for menu recommendation using rough sets. International Journal of Innovative Computing, Information and Control, 7(5(B)):2799-2808, 2011.

[27] Kevin Leyton-Brown and Yoav Shoham. Essentials of game theory: A concise multidisciplinary introduction. Synthesis Lectures on Artificial Intelligence and Machine Learning, 2(1):1-88, 2008.

[28] Stuart E. Middleton, David C. De Roure, and Nigel R. Shadbolt. Capturing knowledge of user preferences: ontologies in recommender systems. In Proceedings of the 1st International Conference on Knowledge Capture. 2001, pp.100107.

[29] Koji Miyahara and Michael J. Pazzani. Collaborative filtering with the simple bayesian classifier. In Proceedings of the Pacific Rim International Conference on Artificial Intelligence. 2000, pp.679-689.

[30] Tomoko Murakami, Koichiro Mori, and Ryohei Orihara. Metrics for evaluating 
the serendipity of recommendation lists. In Proceedings of the Annual Conference of the Japanese Society for Artificial Intelligence. 2007, pp.40-46.

[31] Deuk Hee Park, Hyea Kyeong Kim, Il Young Choi, and Jae Kyeong Kim. A literature review and classification of recommender systems research. Expert Systems with Applications, 39(11):10059-10072, 2012.

[32] Zdzislaw Pawlak. Rough sets. International Journal of Computer 83 Information Sciences, 11(5):341-356, 1982.

[33] Zdzislaw Pawlak. Rough sets and fuzzy sets. Fuzzy Sets and Systems, 17(1):99$102,1985$.

[34] Michael J. Pazzani. A framework for collaborative, content-based and demographic filtering. Artificial Intelligence Review, 13:393-408, 1999.

[35] Michael J. Pazzani and Daniel Billsus. Content-based recommendation systems. In The Adaptive Web. 2007, pp.325-341.

[36] David M. Pennock, Eric Horvitz, Steve Lawrence, and C. Lee Giles. Collaborative filtering by personality diagnosis: A hybrid memory-and model-based approach. In Proceedings of the Sixteenth Conference on Uncertainty in Artificial Intelligence. 2000, pp.473-480.

[37] Yuhua Qian, Hu Zhang, Yanli Sang, and Jiye Liang. Multigranulation decisiontheoretic rough sets. International Journal of Approximate Reasoning, 55(1):225237, 2014.

[38] Al Mamunur Rashid, Istvan Albert, Dan Cosley, Shyong K. Lam, Sean M. McNee, Joseph A. Konstan, and John Riedl. Getting to know you: learning new 
user preferences in recommender systems. In Proceedings of the 7th International Conference on Intelligent User Interfaces. 2002, pp.127-134.

[39] Badrul Sarwar, George Karypis, Joseph Konstan, and John Riedl. Application of dimensionality reduction in recommender system-a case study (no. tr-00-043). Technical report, Minnesota Univ Minneapolis Dept of Computer Science, 2000.

[40] J. Ben Schafer, Dan Frankowski, Jon Herlocker, and Shilad Sen. Collaborative filtering recommender systems. In The Adaptive Web. 2007, pp.291-324.

[41] Andrew I. Schein, Alexandrin Popescul, Lyle H. Ungar, and David M. Pennock. Methods and metrics for cold-start recommendations. In Proceedings of the 25th Annual International ACM SIGIR Conference on Research and Development in Information Retrieval. 2002, pp.253-260.

[42] Vivek Kumar Singh, Mousumi Mukherjee, and Ghanshyam Kumar Mehta. Combining collaborative filtering and sentiment classification for improved movie recommendations. In Multi-disciplinary Trends in Artificial Intelligence. 2011, pp.38-50.

[43] Xiaoyuan Su and Taghi M. Khoshgoftaar. A survey of collaborative filtering techniques. Advances in Artificial Intelligence, 2009:4-23, 2009.

[44] Xiaoyuan Su and Taghi M. Khoshgoftaar. Collaborative filtering for multi-class data using belief nets algorithms. In Proceedings of the 18th IEEE International Conference on Tools with Artificial Intelligence. 2006, pp.497-504.

[45] John A. Swets. Roc analysis applied to the evaluation of medical imaging techniques. Investigative Radiology, 14(2):109-121, 1979. 
[46] Lyle H. Ungar and Dean P. Foster. Clustering methods for collaborative filtering. In Proceedings of the AAAI Workshop on Recommendation Systems. 1998, pp.114-129.

[47] JingTao Yao and Joseph P. Herbert. A game-theoretic perspective on rough set analysis. Journal of Chongqing University of Posts and Telecommunications (Natural Science Edition), 20(3):291-298, 2008. 